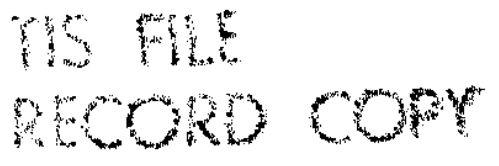

\title{
REACTIVITY EFFECTS \\ OF MODERATOR VOIDS
}

\author{
C. E. AHLFELD \\ R. J. PRYOR
}

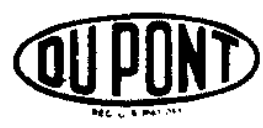

E. I. DU PONT DE NEMOURS AND COMPANY SAVANNAH RIVER LABORATORY AIKEN, SOUTH CAROLINA 29801 


\section{NOTICE}

This report was prepared as an account of work sponsored by the United States Government. Neither the United States nor the United States Department of Energy, nor any of their contractors, subcontractors, or their employees, makes any warranty. express or implied or assumes any legal liability or responsibility for the accuracy, completeness or usefulness of any information, apparatus, product or process disclosed, or represents that its use would not infringe privately owned rights.

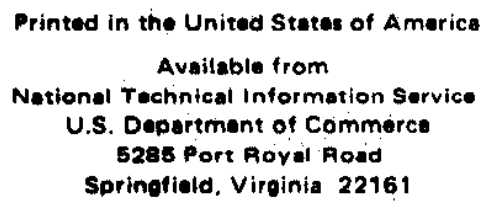

Price: Printed Copy \$6.00; Microfiche \$3.00 


\section{REACTIVITY EFFECTS OF MODERATOR VOIDS}

by

C. E. AHLFELD

R. J. PRYOR

Approved by

J. D. Spencer, Research Manager

Reactor Physics Division

Publication Date: December 1978

ISSUED BY E. I. DU PONT DE NEMOURS AND COMPANY SAVANNAH RIVER LABORATORY AIKEN, SOUTH CAROLINA 29801 


\begin{abstract}
Reactivity worths for large moderator voids similar to those produced by steaming in postulated reactor transients were measured in the Process Development Pile (PDP) reactor. The experimental results were compared to the computed void worths obtained from techniques currently used in routine safety analyses. Two major deficiencies were found. The ce11 calculations used to derive macroscopic cross sections do not take into account the moderated cells surrounding the void, and the diffusion coefficients do not take into account the finite size of the void. These effects led to overpredictions of $30-150 \%$ in $\Delta \mathrm{k}_{\mathrm{eff}}$ for the cases studied.
\end{abstract}

Neutron energy spectrum measurements were used to verify a modified lattice pattern that correctly computed the measured spectrum, and consequent1y, improved macroscopic cross sections. In addition, a special two-dimensional transport calculation was performed to obtain an axially defined diffusion coefficient for the void region. The combination of the modified lattice calculations and the axial diffusion coefficient yielded void reactivity worths which agreed very well with experiment.

It was concluded that the computational modules available in the JOSHUA system (GLASS, GRIMHX) would yie1d accurate void reactivity worths in SRL-SRP safety analysis studies, provided the above mentioned modifications were made. However, the particular cases of embedded voids or partial voids (mixed stean and water) were not addressed in this study, and future investigation of these void types would provide the information needed for further improvements in the computational methods. 


\section{CONTENTS}

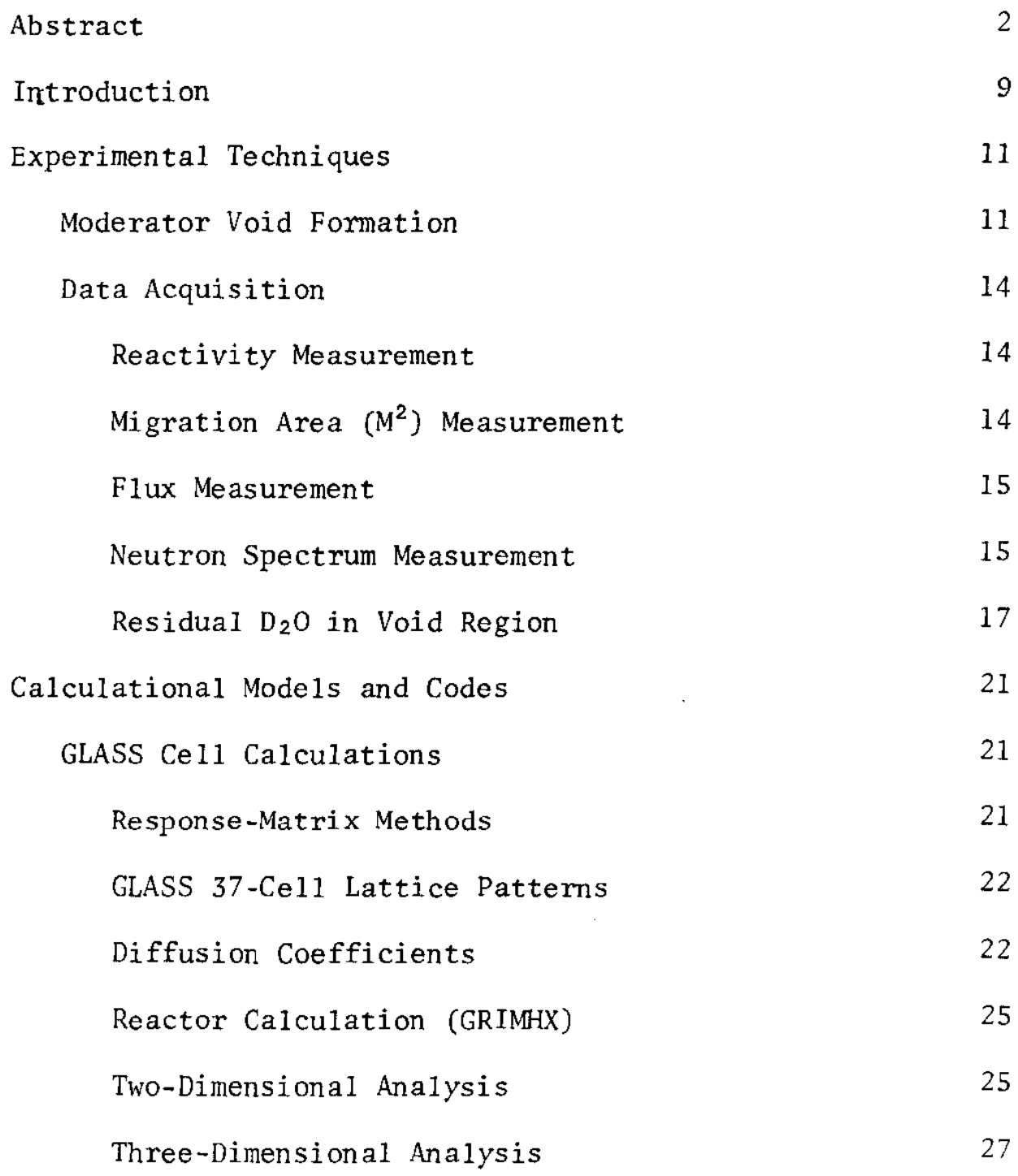


Detailed Results 31

Void Reactivity Worth 31

Enriched Lattice 31

Central Vacancy Lattice 31

Uranium Metal Lattice 36

$\begin{array}{ll}\text { Migration Areas } & 39\end{array}$

Flux Shape Data $\quad 39$

$\begin{array}{ll}\text { Radial F1ux } & 39\end{array}$

Axial Flux 47

Axia1 Leakage Flux $\quad 52$

Neutron Energy Spectra $\quad 52$

Enriched Lattice $\quad 52$

Uranium Metal Lattice 61

$\begin{array}{ll}\text { Conclusions } & 68\end{array}$

$\begin{array}{ll}\text { References } & 69\end{array}$

Appendix

Modified Benoist Method for Diffusion Coefficient 71 Generation for Large Moderator Voids 


\section{LIST OF TABLES}

Table

1

Cross Sections and Resonance Energies for Resonance

20

Foil Detectors

2

Diffusion Coefficients Derived by Isotropic and Anisotropic Methods

3

Detailed Comparison of Measured and Calculated Void Worths

Measured Migration Areas for Moderator Void Test Lattices 


\section{LIST OF FIGURES}

\section{Figure}

1 PDP Void Producing Apparatus for Assemblies in Universa1 Sleeve Housings

2 PDP Gas Handling System for Producing Voids in the Moderator and Coolant Channels

3 Leakage Flux Collimator Showing Schematic Relationship to PDP Reactor

$4 \quad$ Typical Resonance Foil Detector Strip Indicating Location of Various Foils

5 Seven Assembly Void Can Cluster Showing Areas of Residual $\mathrm{D}_{2} \mathrm{O}$ Penetration

$6 \quad$ Normal and Modified Lattice Patterns Employed in GLASS Calculations to Produce Proper Neutron Spectrum

7 Two Region Model for Computing Axial Diffusion Coefficients for Finite Void Cavities

8 Thermal Axia1 Flux Shapes for 3-D GRIMHX with Norma1 and Extended Top Boundaries

9 Side Section View of PDP GRIMHX Model Showing Segment and Mesh Interval Structure

10 PDP Face Map for Enriched Lattice Loading

11 PDP Face Map for Uranium Metal Lattice by Substitution in Enriched Host Lattice

12 Comparison of Measured and Computed Reactivity Change as a Function of Void Length - Enriched Lattice

13 Comparison of Measured and Computed Reactivity Change as 
15 Comparison of Measured and Computed Radial Flux Shapes

- Enriched Lattice - Base Case

16 Comparison of Measured and Computed Radial Flux Shapes

- Enriched Lattice - Voided

17 Comparison of Measured and Computed Radial Flux Shapes

- Central Vacancy Lattice - Base Case

18 Comparison of Measured and Computed Radial Flux Shapes

- Central Vacancy Lattice - Voided

19 Comparison of Measured and Computed Radial Flux Shapes - Uranium Metal Lattice - Base Case

- Uranium Metal Lattice - Voided

21 Thermal Axial Flux Shape Measured and Calculated with GRIMHX - Central Vacancy Lattice

GRIMHX - Uranium Metal Lattice

23 Comparison of Experimental and Theoretical Axial Flux Shapes for Moderated, Partial, and Fully Voided Central Void Region - Enriched Lattice - Thermal

24 Comparison of Experimental and Theoretical Axial Flux Shapes for Moderated, Partial, and Fully Voided Central Void Region - Enriched Lattice - Epitherma1

25 Comparis on of Experimental and Theoretical Axial Flux Shapes for Moderated, Partial, and Fully Voided Central Void Region - Central Vacancy Lattice - Thermal

26 Comparison of Experimental and Theoretical Axial Flux Shapes for Moderated, Partial, and Fully Voided Central Void Region - Central Vacancy Lattice - Epithermal

27 Comparison of Experimenta1 and Theoretical Axial Flux Shapes for Moderated, Partial, and Fully Voided Central Void Region - Uranium Metal Lattice - Thermal

28 Comparison of Experimental and Theoretical Axial Flux Shapes for Moderated, Partial, and Fully Voided Central Void Region - Uranium Metal Lattice - Epithermal 
29 Measured Leakage Flux Data as a Function of Void Length

30 Measured Leakage Flux Data as a Function of Void Length

- Central Vacancy Lattice

31 Measured Leakage Flux Data as a Function of Void Length - Uranium Metal Lattice

32 Comparison of Measured and Calculated Neutron Energy Spectra for the Enriched Lattice at the Center of the Void Region

33 Comparison of Measured and Calculated Neutron Energy Spectra for the Enriched Lattice at the Center of a Voided Fuel Assembly

34 Comparison of Measured and Calculated Neutron Energy Spectra for the Enriched Lattice $5 \mathrm{~cm}$ from Void Interface (in Moderator)

35 Comparison of Measured and Calculated Neutron Energy Spectra for the Uranium Metal Lattice at the Center of the Void Region

36 Compaxison of Measured and Calculated Neutron Energy Spectra for the Uranium Metal Lattice at the Center of a Voided Fue1 Assembly

37 Comparison of Measured and Calculated Neutron Energy Spectra for the Uranium Metal Lattice $5 \mathrm{~cm}$ from Void Interface (in Moderator) 


\section{INTRODUCTION}

Studies of postulated accidents for Savannah River Plant production reactors involving positive reactivity transients have shown that, if shutdown mechanisms do not operate, the transient is initially limited by the formation of steam voids in the hottest assemblies and the surrounding moderator. Consequently, the reactivity worth of these steam voids must be accurately calculated for a meaningful study of the time evolution and ultimate consequences of the postulated transient.

Present SRP accident analysis codes ${ }^{1}$ employ voided cel1 parameters generated by single-cell infinite lattice calculations in which all $\mathrm{D}_{2} \mathrm{O}$ regions are reduced to $1 \%$ of normal density. A series of Process Development Pile (PDP) experiments was conducted to evaluate the present techniques as we 11 as to serve as a test for future code development. Additional objectives of the experiments were to develop remedial techniques if best current calculational methods proved inadequate, and to identify void related problem areas that might be studied in future experiments. The experimental results indicated that the present techniques tend to overestimate void worth in varying degrees from one lattice type to another. The primary calculational errors occurred in the neutron energy spectrum and diffusion coefficient computed with the lattice code GLASS. ${ }^{2}$

A special lattice pattern has been devised which effectively removes the errors in the neutron energy spectrum. The lattice pattern consists of one to seven voided cells surrounded by two complete rings of fully moderated fuel cells. Calculation of accurate diffusion coefficients requires a two-step process which is discussed in detail in subsequent sections. These calculations do not require large scale reprogramming of existing codes. They are similar to those described by Michelini, et al, ${ }^{3}$ but are specifically tailored to reactor codes requiring isotropic diffusion coefficients, such as GRASS. ${ }^{4}$

There are numerous references in the literature pertaining to void effects, the most notable being the methods first proposed by Benoist. ${ }^{5}$ Unfortunately, most of this work is involved with the detailed treatment of neutron "channeling" or "streaming", and does not treat large scale moderator voids encountered during a transient in $\mathrm{D}_{2} \mathrm{O}$ moderated reactors. In general, a directional 
or vectored diffusion coefficient is defined with the result that the component along the axis of a void (e.g., coolant channe1) is increased slightly. It is found that, due to the infinite lattice assumption in GLASS, the increase in the diffusion coefficient for a voided cell is overestimated by as much as a factor of ten. A more rigorous calculation is required which removes the infinite lattice assumption and accounts for the void volume. 
EXPERIMENTAL TECHNIQUES

\section{Moderator Void Formation}

In order to measure the reactivity effects of moderator voids, a reliable and reproducible means of creating voided cavities in both the coolant and the moderator regions for selected fuel assemblies had to be devised. Voids were obtained by means of hexagonally shaped aluminum cans enclosing the fu11 length of a fuel assembly. These void cans (Figure 1) were fabricated and installed around the center seven assemblies of three different PDP lattice types. The void can and universal sleeve housing (USH) are fitted with separate gas inlets orificed to 0.062 in. This feature allows the helium gas to enter or release slowly, thus preventing sudden changes in reactivity (typical max $k \approx 0.15 \$ / \mathrm{sec}$ ). Helium gas pressurization is remotely controlled from the PDP control room with the reactor at critical (Figure 2). Simultaneously, the PDP reactor operator adjusts the $\mathrm{D}_{2} \mathrm{O}$ height to just offset the negative worth of the void. The action is repeated incrementally until the desired voiding

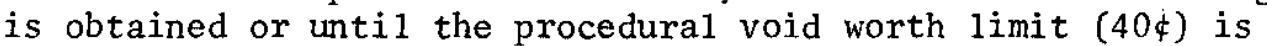
reached.

The depth of the void in each assembly was determined by high-precision pressure readings from a folded, oil-filled manometer. These readings were reproducible to approximately $1 \mathrm{~mm}$ of $\mathrm{D}_{2} \mathrm{O}$ height. A secondary depth indicator in the form of a dip tube located at the PDP tank wall also served as a safety device. The dip tube was pressurized by the same helium manifold as the void cans. The tube was attached to a control rod motor drive and calibrated tape. The dip tube was constructed in such a way as to ensure the bottom open end could never be driven below the bottoms of the void cans. Therefore, in the case of overpressurization, bubbling occurs only at the tank wall with no reactivity effect. The dip tube also functioned as a height indicator. The tube was driven down until it touched the surface of the water and this setting was used as a reference. Direct readings from this drive motor tape gave a good measurement of the void depth. 


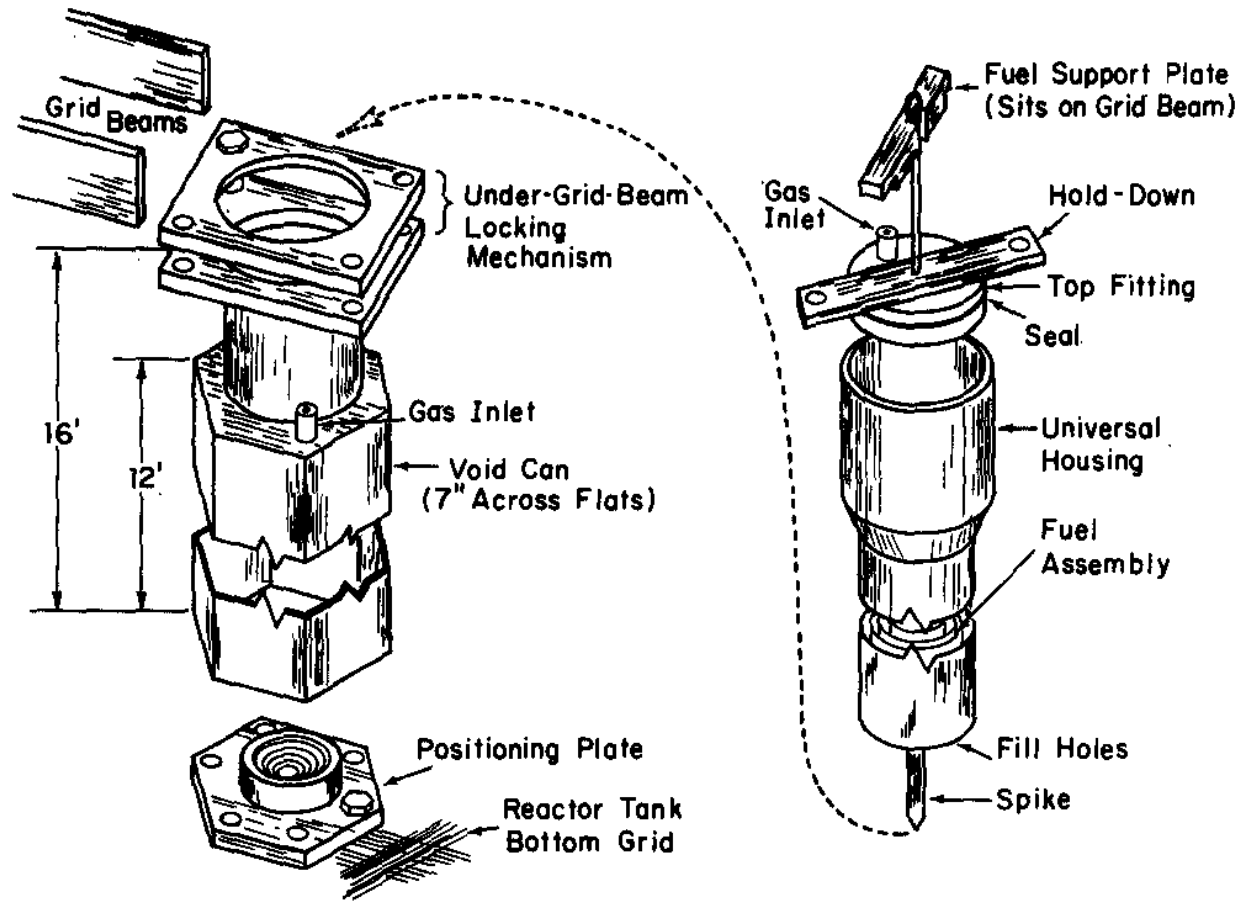

FIGURE 1. PDP Void Producing Apparatus for Assemblies in Universal Sleeve Housings 


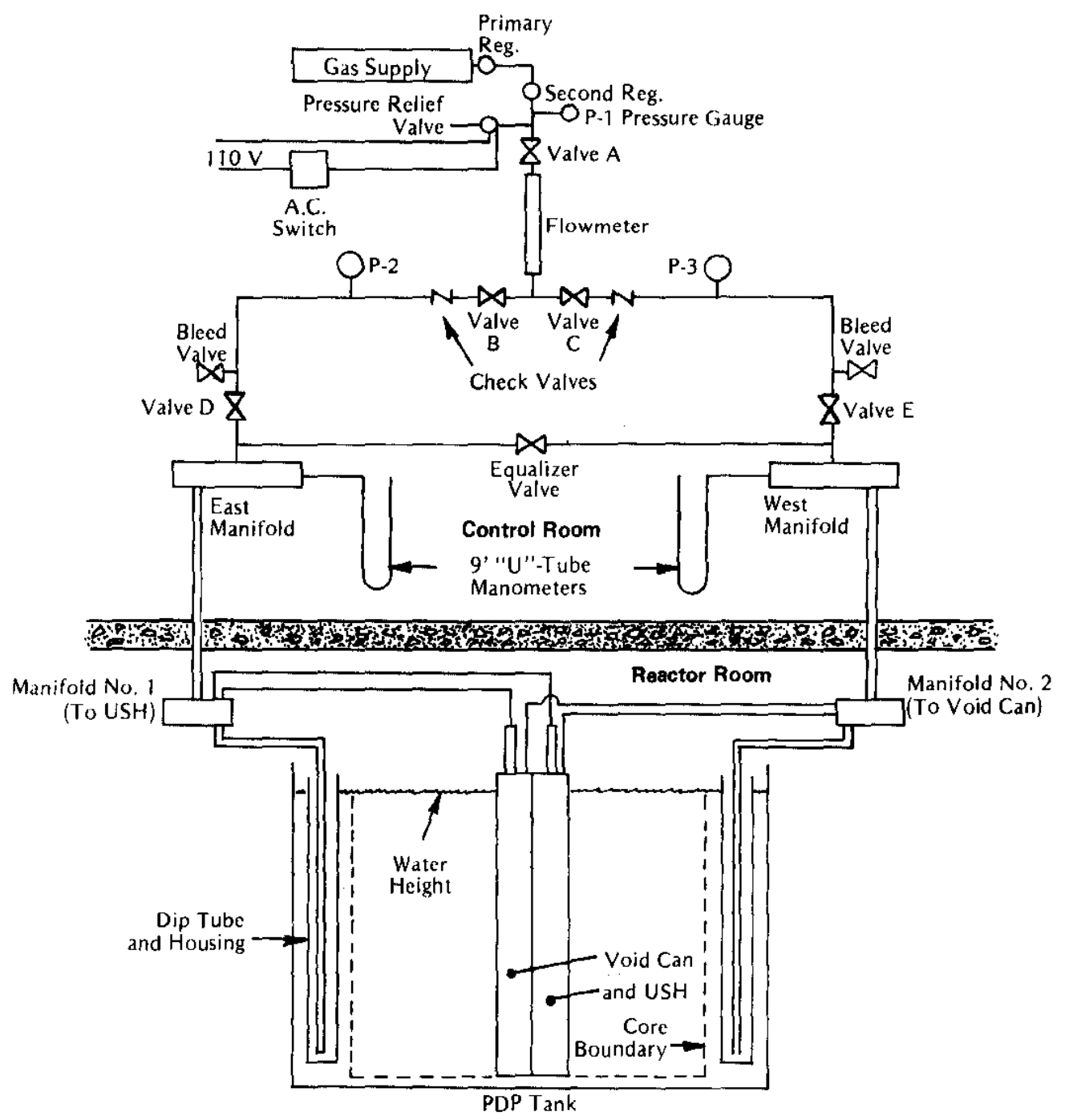

FIGURE 2. PDP Gas Handling System for Producing Voids in the Moderator and Coolant Channels 
Data Acquisition

\section{Reactivity Measurement}

Al1 reactivity measurements were made using the electronic water height sensor to determine the relative $D_{2} O$ level to $\pm 0.05 \mathrm{~mm}$. Changes in $\mathrm{D}_{2} \mathrm{O}$ level are easily related to axial buckling changes since the PDP has a right circular cylindrical geometry. The axial buckling changes, $\Delta B_{z}{ }^{2}$, are related to reactivity worth by the expression

$$
\frac{\Delta \mathrm{k}(\boldsymbol{\phi})}{\mathrm{k}}=\left(1 / \mathrm{B}_{\text {eff }}\right) \frac{\mathrm{M}^{2} \Delta \mathrm{B}_{\mathrm{z}}{ }^{2}}{1+\mathrm{M}^{2} \mathrm{~B}_{\mathrm{g}}{ }^{2}}
$$

$M^{2}$ is the migration area (see the following section)

$\mathrm{Bg}^{2}$ is the geometric buckling

$\beta_{\text {eff }}$ is the effective delayed neutron fraction

Sources of uncertainty and the various corrections applied to this type of data are discussed on page 39.

\section{Migration Area $\left(M^{2}\right)$ Measurement}

The migration area, $\mathrm{M}^{2}$, is a crucial parameter in the reactivity equation above, and for this reason measurements of $\mathrm{M}^{2}$ were obtained by means of asymptotic period data. Initially, the reactor was taken critical at low power in the same configuration which served as the base case for the void reactivity measurements. After delayed neutrons had equilibrated, a small $(\sim 2-3 \$)$ but precisely measured amount of reactivity in the form of $\mathrm{D}_{2} \mathrm{O}$ was added and offset by control rod insertion. Again after delayed neutron equilibration, the control rods were driven out, putting the reactor on a positive period. A Nuclear Data-2200 multichannel analyzer was used to acquire and store neutron detector counts as a function of time after the rod pull. The in-core neutron detectors were recorded until the PDP reached its operating power limit, at which point the operator scrammed the reactor. By assuring an asymptotic behavior, point reactor kinetics should give an exact solution for the time dependent flux in the reactor. The period data were fitted by using $\mathrm{M}^{2}$ as the adjustable fitting parameter in a point reactor kinetics calculation.

This same experimental procedure was repeated for the case of the maximum void configuration. $\mathrm{M}^{2}$ data for intermediate voids was linearly interpolated between these two end-point measurements. 


\section{Flux Measurement}

As in most extensive experimental reactor programs, radial and axial flux shapes were measured to aid in the evaluation and optimization of static reactor computations. The standard gold pin irradiation technique was employed where high purity $(>99.9 \%)$ gold in the form of 0.062 -in-dia by $0.500-i n-10 n$ pins, both bare and under $30 \mathrm{mil}$ cadmium covers, are inserted in equivalent interstitial positions. "Equivalent positions" indicates that the nearest neighboring assemblies are all of the same type (e.g., three drivers, etc.). Because the neutron spectrum is the same for all pins (except in the vicinity of voids where corrections are required), the relative flux shapes are easily obtained by counting the $411.8 \mathrm{kev}$ gamma activity in the irradiated pins with an automatic sample changer and data acquisition system.

Although the gold pin technique is a very effective method for flux mapping, it requires one high-power irradiation for each map. This requirement would have made detailed mapping of the various void configurations tedious and time consuming. Therefore, a boron-lined traveling flux monitor was used for axial flux scans in and near the void region. Thus, in a single reactor run, axial flux data were obtained for all void configurations.

It was anticipated that difficulties might arise in the generation of accurate diffusion coefficients for the void region. One way of directly detecting the effects of changing diffusion coefficients would be to observe the flux leaking only from the void region. This type of leakage is obtained from the $\mathrm{DB}^{2} \phi$ leakage term of diffusion theory. In order to measure this effect, a neutron collimator was constructed from a mixture of boron carbide and polyester resin. The collimator was surrounded by an annulus of paraffin. The central aperture (Figure 3) permitted a 1-in.-dia $\mathrm{BF}_{3}$ neutron detector to be remotely raised or lowered in the collimator. The collimator was positioned directly over the center voided assembly and carefully aligned on the vertical axis of the center assembly. Background tests were made with a 24-in. lucite rod and 20-mi1 cadmium cover placed in the detector channel. Backgrounds were very small, typically 0.1 to $0.5 \%$ of the total thermal leakage counts, and 0.5 to $2.0 \%$ of the total epicadmium leakage counts.

\section{Neutron Spectrum Measurement}

Voiding of coolant and moderator regions causes a spectral shift to neutrons of relatively higher energies. This shift is grossly overestimated in cell or small supercell calculations because the moderating effect of the unvoided remainder of the 


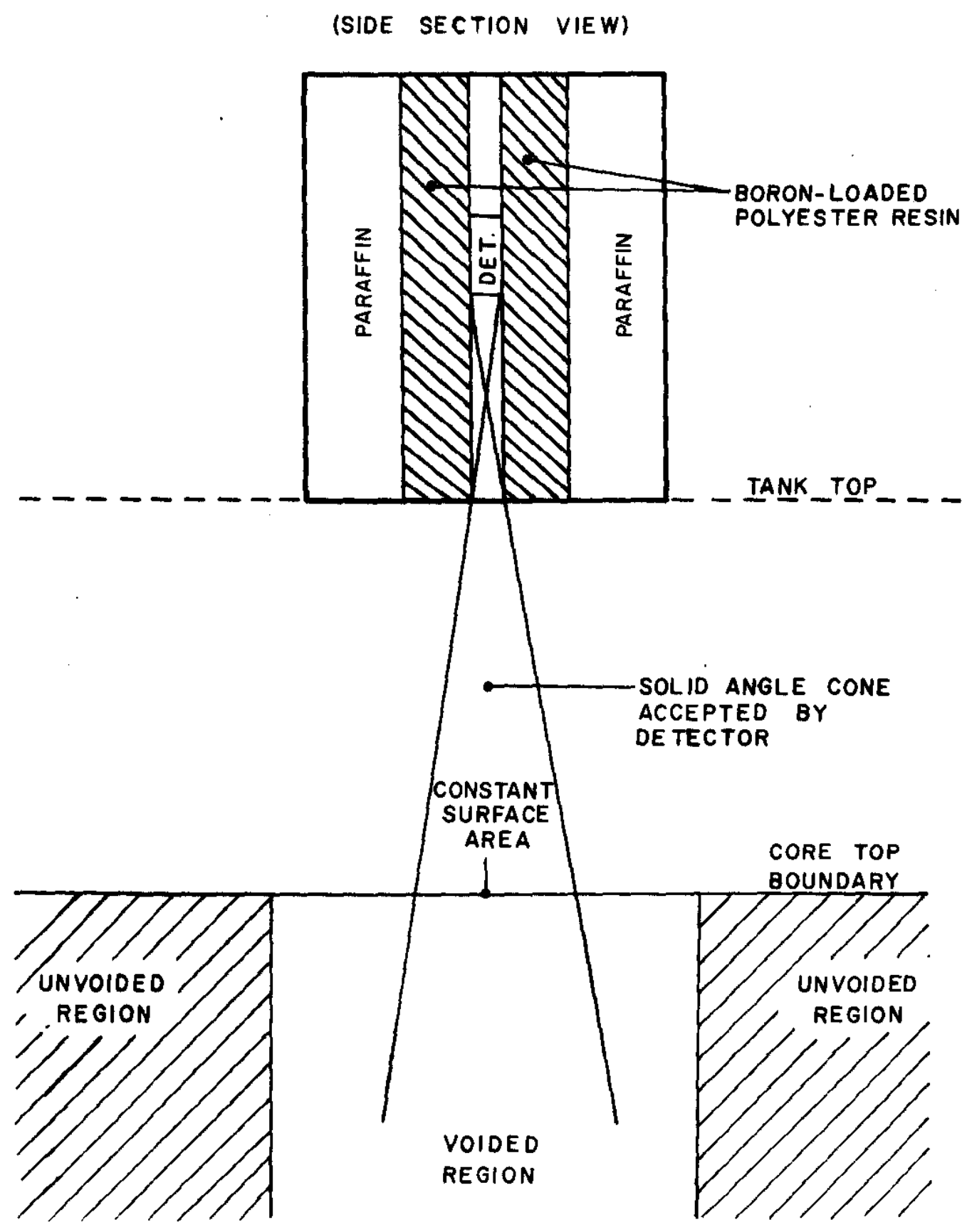

FIGURE 3. Leakage Flux Collimator Showing Schematic Relationship to PDP Reactor 
reactor is not taken into account. Neutron spectrum measurements were performed to provide a basis for spectral correction techniques. Resonance foil detectors (Table 1) were used to measure the spectrum in voided fuel assemblies, interstitial positions, voided vacant assemblies, and in the moderator region adjacent to the voids. This technique is applicable only to those nuclides which have a single strong resonance in the energy region of interest. Furthermore, if relative counting techniques are to be used, a thermal reference spectrum must be established, and a similar set of foils must be simultaneously irradiated in this thermal spectrum. For the PDP measurements, a septifoil in Gang III was removed to provide a thermal reference point. This position gave a ${ }^{235} \mathrm{U}$ cadmium ratio of 228.4 which is not adequately thermalized, but could be effectively corrected by subsequent cell calculations. Figure 4 shows a typical foil stringer as it was placed in the reactor test position. Note that copper pins were positioned along the length of the strip in order to normalize the axial flux variations over the length of the strip, as well as from one strip to another where more than one strip was placed at the same reactor location. The irradiated foils were counted with an automatic sample changer system, but due to half-life and cross section differences, foils of a given type were counted at different time intervals after the irradiation. The gamma activity counted was also obtained at different energy threshold and window settings to enhance true signal-to-background ratios. The normalization and various corrections applied to the data are described on page 39.

\section{Residual $\mathrm{D}_{2} \mathrm{O}$ in Void Region}

Void cans were fabricated by bending 0.060-in.-thick 6061 aluminum sheet into two half-hex shells on a forming brake. The two halves were welded and the seams were ground to permit close clustering of up to seven such hexagonal assemblies (Figure 5). Machine tolerances and subsequent handling of the assemblies caused small spaces between adjacent hexagonal flats; these spaces contained small amounts of $\mathrm{D}_{2} \mathrm{O}$ during voided reactor experiments. In order to obtain an estimate of the amount of $\mathrm{D}_{2} \mathrm{O}$ residing in these gaps, "feeler" gauges were inserted along the length of the cluster at 12-inch intervals in the six accessible gaps. The center assembly could not be gauged so the average of the six gaps directly measured were applied to the appropriate gaps in the center assembly. The results of these measurements gave a residual $\mathrm{D}_{2} \mathrm{O}$ content of approximately $1.3 \%$ when distributed over the entire void region. Since all of the void calculations to be described later replace norma $1 \mathrm{D}_{2} \mathrm{O}$ moderator and coolant with $\mathrm{D}_{2} \mathrm{O}$ having $1 \%$ of normal density, this residual $\mathrm{D}_{2} \mathrm{O}$ brings the calculation closer to the actual case measured in the reactor. 


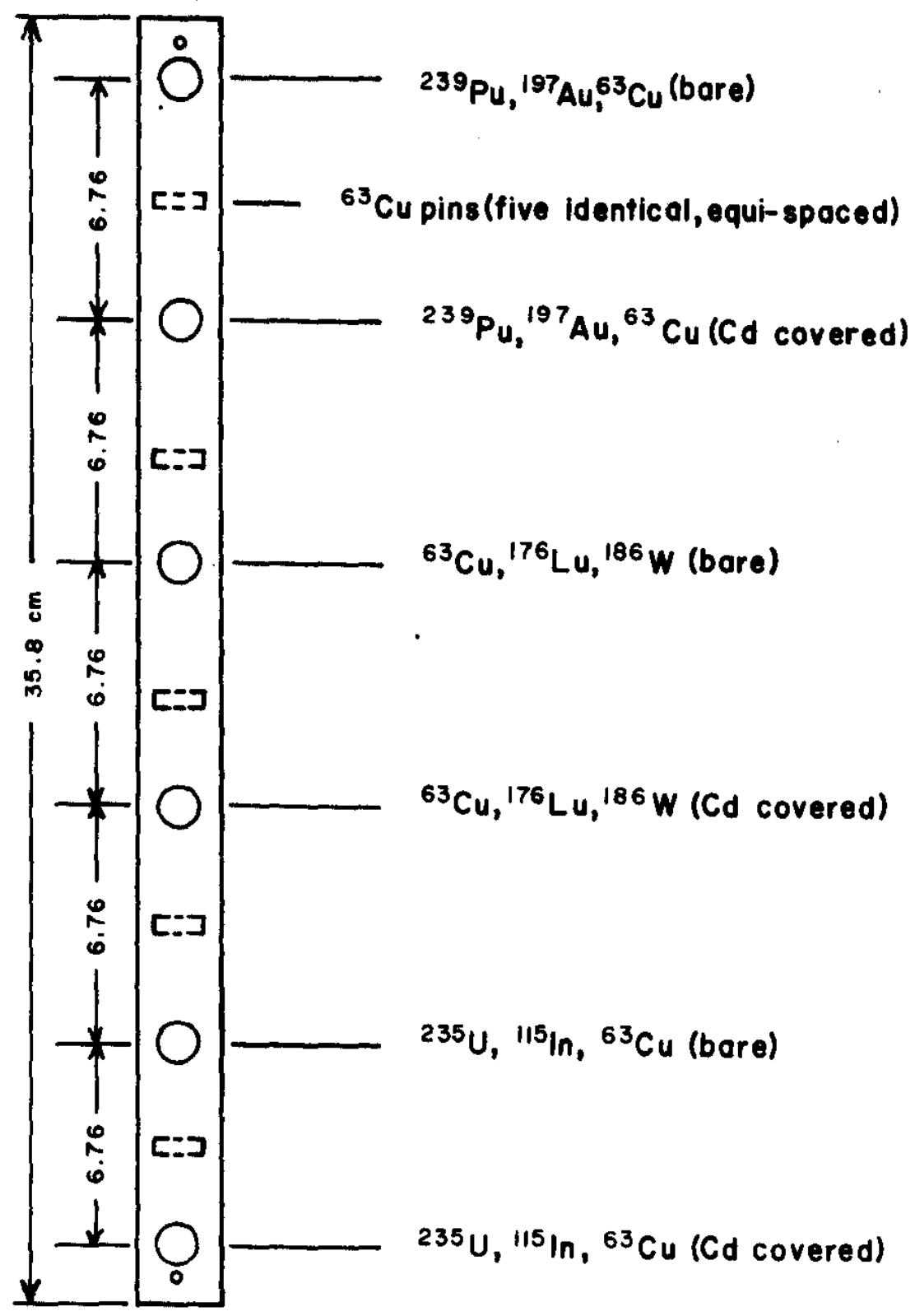

FIGURE 4. Typical Resonance Foil Detector Strip Indicating Location of Various Foils 


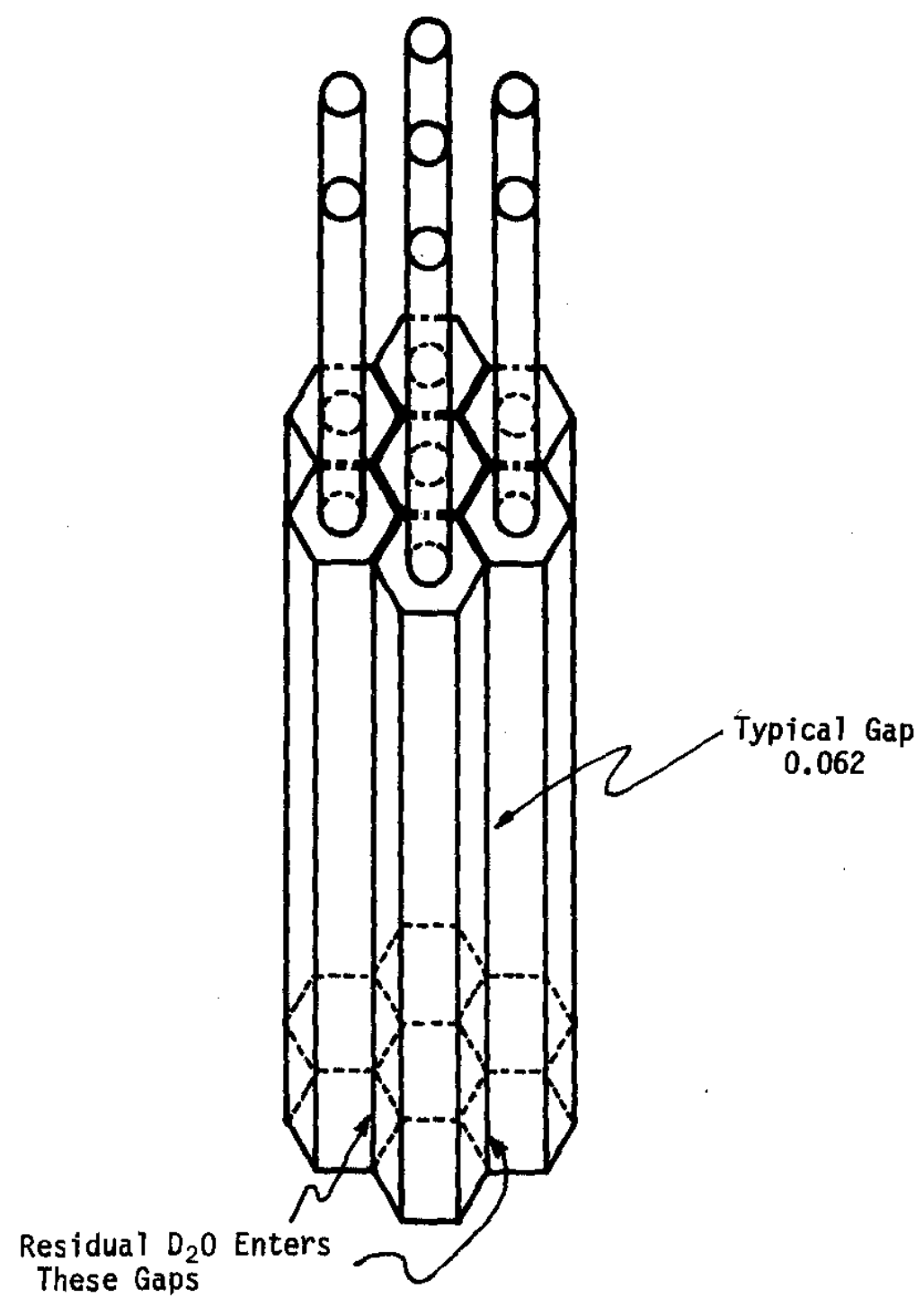

FIGURE 5. Seven Assembly Void Can Cluster Showing Areas of Residua] $\mathrm{D}_{2} \mathrm{O}$ Penetration 
TABLE 1

Cross Sections and Resonance Energies for Resonance Foil Detectors

$\begin{array}{lcclc}\text { Isotope } & \begin{array}{l}\text { Resonance Energy, } \\ \text { electron volts }\end{array} & \begin{array}{l}g \sigma_{2200,} \\ \text { barms }\end{array} & \begin{array}{l}\text { Resonance } \\ \text { Integral, } \\ \text { barms }\end{array} & \begin{array}{c}\text { oeffa } \\ \text { barns }\end{array} \\ { }^{176} \mathrm{Lu} & 0.142 & 3080.6 & 8346 \mathrm{~b} & 5872 \\ { }^{2{ }^{9} \mathrm{Pu}} & 0.296 & 740.9 & 1626^{b} & 894.4 \\ { }^{115} \mathrm{In} & 1.46 & 195.0 & 2550 & 201.4 \\ { }^{197} \mathrm{Au} & 4.91 & 99.4 & 1490 & 104.3 \\ { }^{186} \mathrm{~W} & 18.8 & 38.0 & 410^{c} & 38.1 \\ { }^{63} \mathrm{Cu} & 577 & 4.5 & 2.04(+1.82)^{d} & 4.859\end{array}$

a. Corrected for partially thermalized reference position.

$b$. Derived by numerical integration of differential cross section over desired lethargy range.

c. Includes effect of overlapping $\mathrm{Cd} 18.5 \mathrm{eV}$ resonance.

d. $1.82 \mathrm{~b}$ smooth $(\eta l / v)$ cross section integra1. 


\section{CALCULATIONAL MODELS AND CODES}

\section{GLASS Cell Calculation}

The infinite 1attice transport theory code, GLASS (formerly RAHAB) was used to generate fewgroup cell parameters for both normal and voided assemblies for subsequent reactor calculations. Using single cell or small supercell lattice patterns ( 2 or 3 unique assemblies) resulted in void cell diffusion coefficients which were too large by as much as a factor of 10 . In addition, the 37-group neutron energy spectrum for voided assemblies was much too hard, peaking in the kilovolt energy region. Both of these effects result from the infinite repeating nature of the lattice pattern which does not correctly model the unvoided region of the reactor. One approach to correct this model deficiency would be to use a coupled-cell calculation which would permit a more exact model of the reactor.

\section{Response-Matrix Methods}

Response-matrix calculations ${ }^{6,7}$ (RESPONSE) appear to offer a potential solution to the problem identified in GLASS in the previous section. The response technique calculates the response of a single cell to a neutron current entering through one of its faces. This is achieved by a combination of Monte Carlo and collision probability methods with the results expressed in the form of response matrices for each cell instead of cross sections as is the case with GLASS. The global reactor calculation (RASCAL) is accomplished by coupling the neutron partial currents throughout the reactor. Thus, the effects of adjacent cells are more exactly represented since there is no infinite lattice assumption made.

Despite the relative ease and accuracy of reactor modeling, the method suffers from several disadvantages with respect to voided reactor computations. Chief among these is its limitation to two-dimensional reactor calculations. In the PDP void experiments, bottom reflector and top boundary extrapolation are crucial features for accurate reactivity worth calculations. Top and bottom region modeling can be accomplished only with a full threedimensional reactor calculation. Since RESPONSE does not calculate detailed fluxes over the cell, the neutron spectrum is not available for comparison with its experimental equivalent. Finally, in comparative GLASS/GRIMHX and RESPONSE/RASCAL 2D studies, the reactivity effects due to voids was found to be very similar and not in agreement with experiment. 


\section{GLASS 37-Cel1 Lattice Patterns}

In order to obtain region-averaged cross section data for 3-D diffusion theory calculations, a method was devised which overcame the hard spectrum difficulties of single or small superce11 GLASS computations. A new lattice pattern was created which permitted a single cluster of 37 cells composed of seven unique cells (Figure 6). For seven voided assemblies, this new pattern allows two additional rings of fully moderated assemblies to surround the void, thus softening the spectrum to agree better with experiment. This pattem gave good spectral representations for all lattices and test locations studied, and is expected to have general applicability to all SRP/SRL reactor calculations where moderator voiding is present. Experimental spectral data and 37-cell GLASS spectra are illustrated on pages 62-67.

\section{Diffusion Coefficients}

As stated earlier, the reactor calculation (GRASS) overestimates axial leakage when the diffusion parameters are computed with GLASS (including 37-ce11 lattice patterns). This overestimate stems from the fact that the diffusion coefficient, or more properly $1 /\left(3 \Sigma_{\mathrm{T}}\right)$, is calculated isotropically on a cell by cell basis. Consequently, GLASS does not accurately model the finite size of the reactor void with respect to the rest of the reactor.

Anisotropic diffusion effects are generally represented by radial and axial diffusion coefficients. In this study, a twodimensional, two-region transport calculation was used to compute axial leakage out of a cylindrically shaped void region in the reactor. The computed leakage is equated to the familiar $\mathrm{DB}_{z}{ }^{2} \phi$ leakage term of diffusion theory. Figure 7 illustrates the geometric model and transport equation used to compute this "axial" diffusion coefficient. In this equation, $\mathrm{B}_{z}{ }^{2}$ is measured in this experiment, and the $\Sigma_{\mathrm{a}}$ and $\Sigma_{\mathrm{S}}$ values are obtained from the previously described GLASS calculations. A cosine flux distribution is assumed over the height of the reactor. This procedure has been coded under the module name LEAK and is executed via the JOSHUA ${ }^{2}$ system. Computer CPU time typically runs from 15 to 30 minutes per problem which would be intolerable in routine safety analyses. However, the size of the correction on $D$ varies smoothly as a function of void radius, and therefore, the correction is accurately computed by a polynomial expansion representation. Recent1y, Graves (see Appendix) has suggested an alternative method which yields approximately the same axial diffusion coefficient, but requires significantly less computer time. 


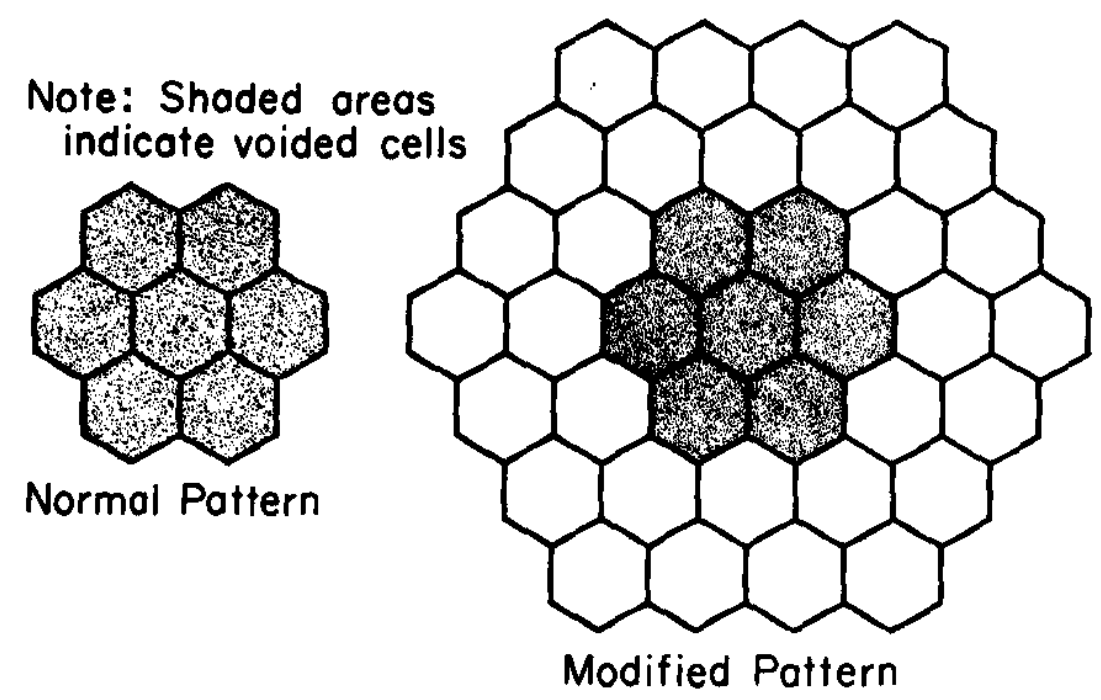

FIGURE 6. Normal and Modified Lattice Patterns Employed in GLASS Calculations to Produce Proper Neutron Spectrum 


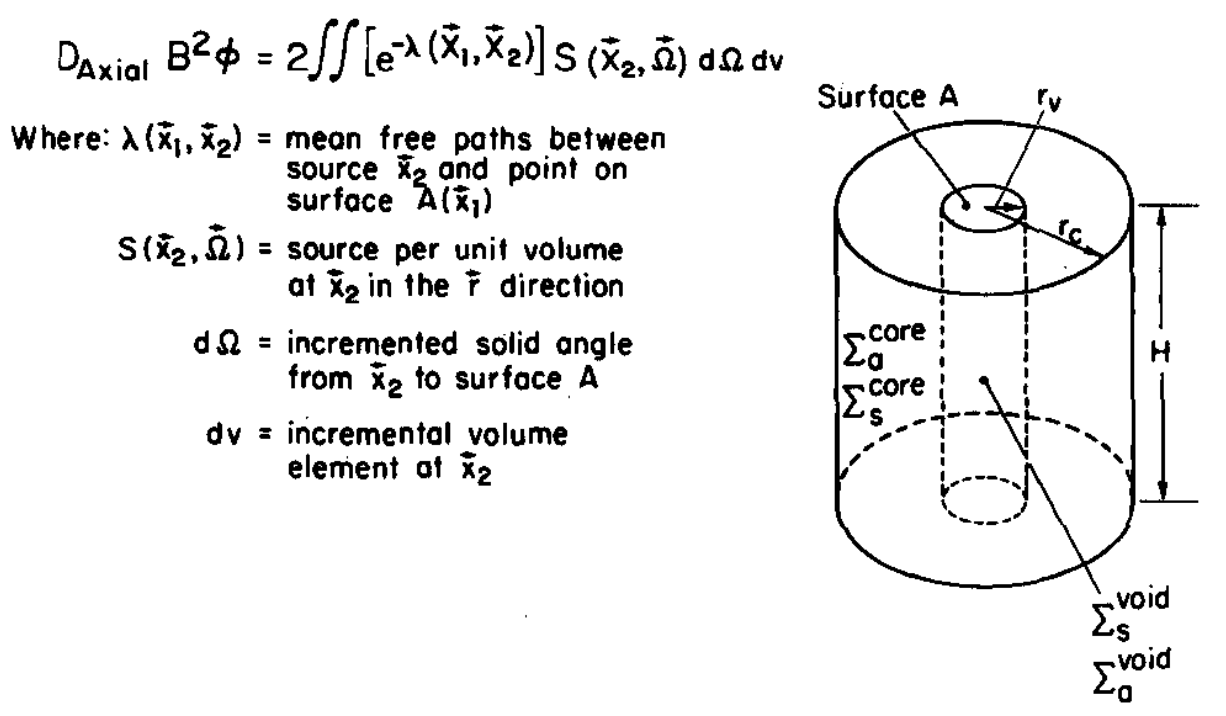

FIGURE 7. Two Region Model for Computing Axial Diffusion Coefficients for Finite Void Cavities 
With either method, the use of an axially defined diffusion coefficient in an isotropic diffusion theory calculation must be justified. In the calculation, the radial component is set equal to $D_{\text {axial }}$. To check the validity of this procedure, a series of axially infinite (i.e., no leakage) calculations were run with both isotropic and equivalent axial diffusion coefficients. The results were identical within the uncertainties of the input parameters. This implies that for voids which are small compared to the rest of the reactor, radial leakage is insensitive to the diffusion coefficients in the void region. As the void region gets very large (of the same order as the reactor), the isotropic result (GLASS) is obtained. Therefore, direct insertion of axial diffusion coefficients in the void region appears justified. A summary of the diffusion coefficients obtained by the various techniques for the three basic lattice types studied is given in Table 2 .

\section{Reactor Calculation (GRIMHX)}

GRIMHX ${ }^{4}$ is a two- or three-dimensional neutronics module in GRASS. GRASS is a generalized diffusion theory reactor calculation within the SRL JOSHUA system. The code has a variety of symmetry options and mesh point assignments. Unless otherwise noted, all calculations were performed in hexagonal geometry for a $60^{\circ}$ sector of the reactor with three mesh points per hex. Cross section data were input as supercell averages for the unperturbed regions of the reactor and as cell averages for the voided regions.

\section{Two-Dimensional Analys is}

Initially, a two-dimensional, hexagonal geometry was selected using the measured axial buckling. Extrapolated top and bottom boundaries were inferred from cosine fits to measured axial flux shapes. The base case calculation (no voids) was computed with uniformly adjusted $\nu \Sigma_{f}$ parameters to yield $k_{e f f}$ exactly equal to unity. This adjustment is necessary because of uncertainties in the ${ }^{6} \mathrm{Li}$ content of the target tubes and, to a lesser extent, because of uncertainties in the ${ }^{235} \mathrm{U}$ content of the fuel tubes. Changes in $\nu \Sigma_{f}$ were typically less than $0.5 \%$ of that computed by GLASS. For the voided calculations, the spectrum-corrected cross sections and LEAK-computed diffusion coefficients were used in the void region. $\Delta \mathrm{k}$ worth of the void is simply the difference between the base and void case eigenvalues.

Void worths computed using a two-dimensional model were not in good agreement with experiment; calculated values were 30 to $150 \%$ greater than the measured values. A significant portion of 
TABLE 2

Diffusion Coefficients Derived by Isotropic and Anisotropic Methods

$\begin{aligned} & \text { PDP } \\ & \text { Charge Type } \\ & \text { Enriched Lattice } \\ & \text { (Normal Moderation) } \\ & \text { Enriched Lattice } \\ & \sigma \quad \quad \quad \text { (7-cell Voided) } \\ & \text { Uranium Metal Lattice } \\ & \text { (Normal Moderation) } \\ & \text { Uranium Metal Lattice } \\ & \text { (7-cel1 Voided) } \\ & \text { Central Vacancy Lattice } \\ & \text { (Normal Moderation) } \\ & \text { Central Vacancy Lattice } \\ & \text { (7-cell Voided) }\end{aligned}$

$\begin{array}{ll}\text { DIFFUSION COEFFICIENT, D } \\ \begin{array}{l}\text { Energy } \\ \text { Group }\end{array} & \begin{array}{l}\text { GLASS-Normal Pattem } \\ \text { (Isotropic) }\end{array}\end{array}$
GLASS-37 CeII LEAK (2 region reactor) (Isotropic) (Anisotropic)

$\begin{array}{rccl}\text { I } & 1.382 & 1.361 & -- \\ \text { II } & 0.8980 & 0.8724 & -- \\ \text { I } & 15.86 & 14.34 & 9.854 \\ \text { II } & 9.921 & 8.292 & 4.026 \\ \text { I } & 1.255 & 1.214 & -- \\ \text { II } & 0.8786 & 0.8449 & -- \\ \text { I } & 5.745 & 6.108 & 6.074 \\ \text { II } & 6.758 & 6.619 & 4.595 \\ \text { I } & 1.279 & 1.250 & -- \\ \text { II } & 0.8669 & 0.8281 & -- \\ \text { I } & 62.53 & 71.31 & 13.98 \\ \text { II } & 75.32 & 61.71 & 11.82\end{array}$


this discrepancy is due to the treatment of top and bottom boundaries in the PDP. The bottom is bounded by approximately $50 \mathrm{~cm}$ of $\mathrm{D}_{2} \mathrm{O}$ reflector with control rods extending to the tank bottom. At the top boundary, the fuel extends beyond the critical $\mathrm{D}_{2} \mathrm{O}$ height, and leakage neutrons interact with this fuel to produce significant neutron feedback. However, the greatest inaccuracy in the two-dimensional (2-D) mockup is simply that, during the experiments, the void region did not extend to the tank bottom. Structural design fixed the maximum void configuration to a depth approximately $30 \mathrm{~cm}$ from the bottom of the tank. This $30 \mathrm{~cm}$ "void reflector" greatly reduces neutron leakage that was computed in the 2-D calculation. Although various corrections and estimates for these effects could be applied to the 2-D calculation, an exact solution is possible with a three-dimensional (3-D) model of the reactor.

\section{Three-Dimensional Analys is}

The three-dimensional option in GRIMHX employs a hex-Z geometry which yields an accurate model of the PDP for the void studies. The $Z$-axis was divided into sixteen segments, and mesh points were assigned at approximately $10 \mathrm{~cm}$ levels. By placing the $\mathrm{Z}$-segment boundaries at the same levels that void steps were taken in the experiment, it was possible to model the void region incrementally in the calculation just as it occurred in the experiment. As expected, agreement with experiment was greatly improved over the $2-D$ results.

However, even after proper treatment of the bottom reflector both in and out of the void region, a significant discrepancy still existed. The nature of the difficulty was diagnosed by the axial flux shape data. Figure 8 shows the axial flux data from a set of gold pins irradiated in Gang III. The dashed curve was calculated ignoring any reactor components above the critical $\mathrm{D}_{2} \mathrm{O}$ height. The solid curve is the same calculation except that an additional $Z$-segment has been added to take into account the fuel extending above the $\mathrm{D}_{2} \mathrm{O}$. For this extended top segment, void region cross sections and diffusion coefficients were used. Figure 9 illustrates the segmented $Z$-axis structure of the final 3-D geometry employed in the analysis. Although this extended top segment technique is not required for SRP production reactors, it is significant in most, if not al1, PDP 3-D calculations where precision buckling, critical water height, or reactivity information in concerned. The authors strongly recommend that future PDP calculations include this technique. 


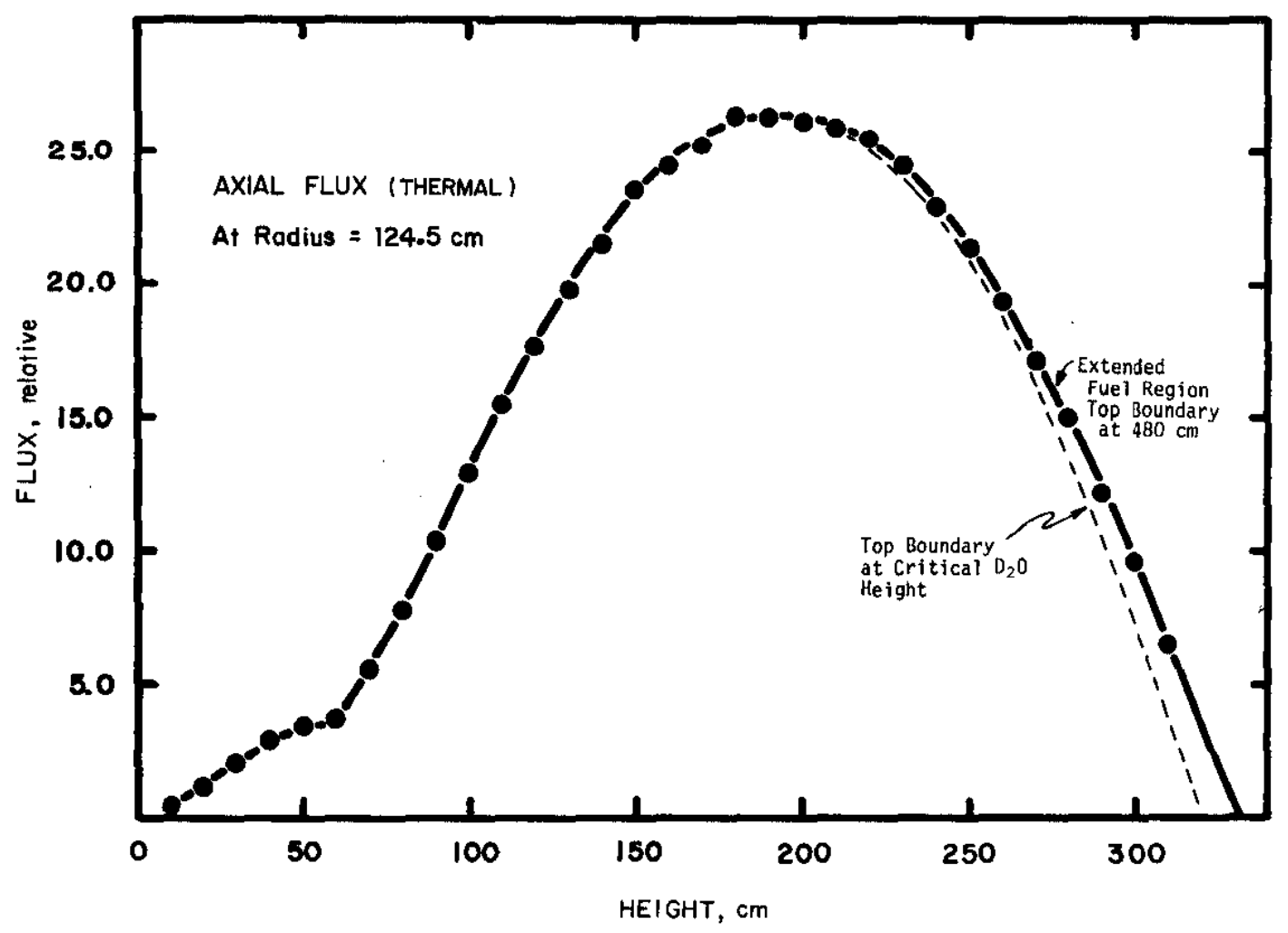

FIGURE 8. Thermal Axial Flux Shapes for 3-D GRIMHX with Normal and Extended Top Boundaries 


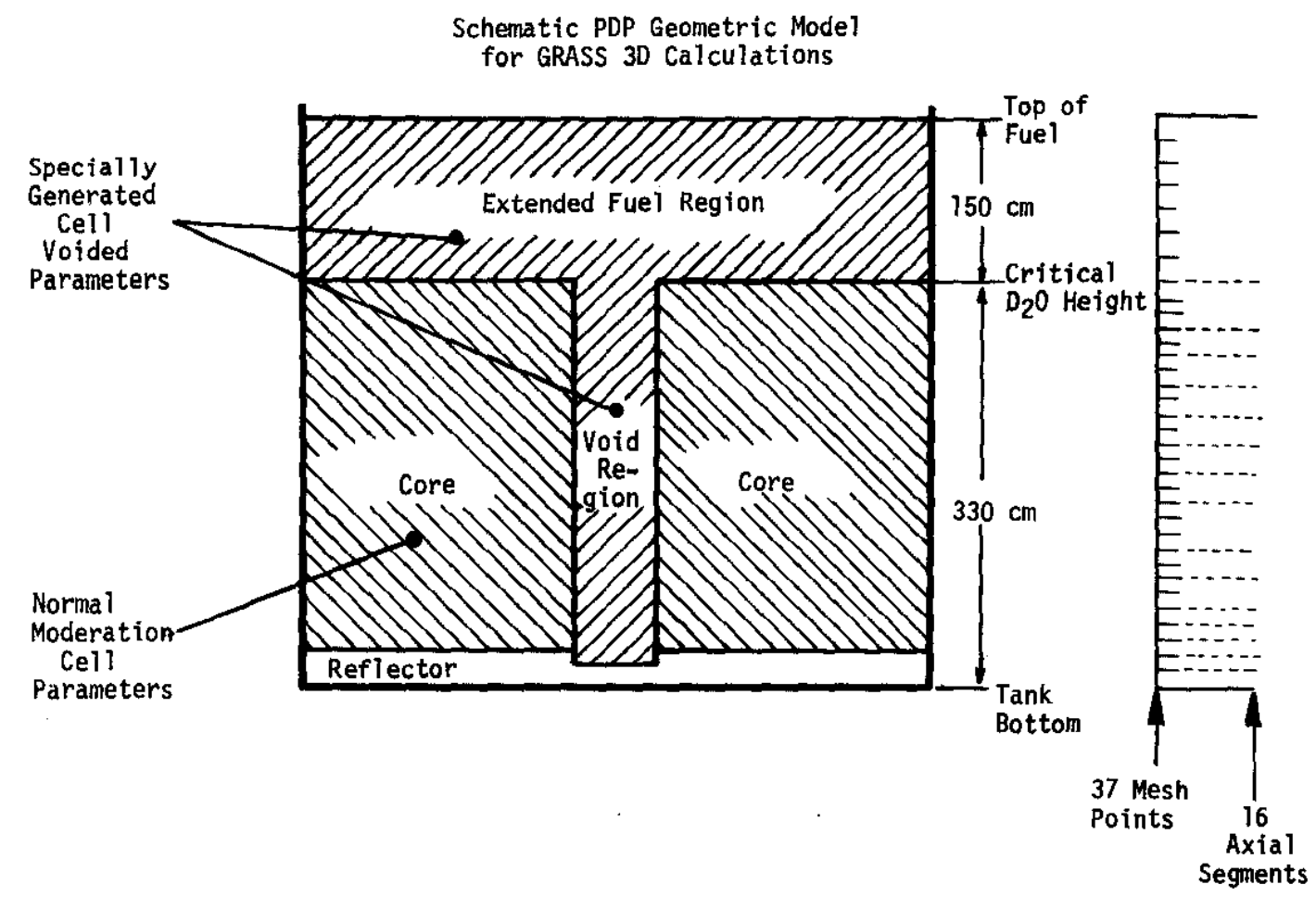

FIGURE 9. Side Section View of PDP GRIMHX Model Showing Segment and Mesh Interval Structure 
$-30-$ 


\section{DETAILED RESULTS}

The measurements described on pages 14-17 were applied to three distinctly different reactor lattice loadings. The first lattice type (hereafter referred to as Enriched Lattice) was a uniform (7" triangular pitch) loading of highly enriched ${ }^{235} \mathrm{U}$ aluminum alloy assemblies. The fuel assemblies contained two concentric fuel tubes and two ${ }^{6} \mathrm{Li} / \mathrm{Al}$ target tubes nested within an aluminum outer housing. Figure 10 illustrates a face map for this lattice.

A second lattice type was constructed by removing the fue 1 assemblies from the void region. The result was to obtain a voidable region without neutron production or significant absorption. Thus, voids in this region will affect reactivity almost entirely by neutron leakage. In the remainder of the discussion, this lattice will be referred to as the Central Vacancy Lattice.

A third lattice configuration was obtained from the basic Enriched Lattice by substituting uranium metal fuel having near natura $1{ }^{235} U$ content, for the enriched assemblies in the center 42 positions including the six fuel positions in the void region. The result (Figure 11 ) is a substitution lattice which permits void studies of a near natural uranium metal lattice without the need for an entire lattice change. This lattice will be designated the Uranium Metal Lattice.

\section{Void Reactivity Worth}

\section{Enriched Lattice}

Measured void worths for the voiding of seven assemblies in the Enriched Lattice are shown in Figure 12. Reactivity is in Beff units ( $\phi$ ) and void length is measured with respect to the top (critical $\mathrm{D}_{2} \mathrm{O}$ height) of the reactor. The error bars assigned to the data points include uncertainties in critical $\mathrm{D}_{2} \mathrm{O}$ height, void length, and migration area (see later section). The solid curve is a GRIMHX 3-D calculation which agrees very well with the experimental data. The corresponding 2-D calculations using spectrum corrected cross sections and proper axial diffusion coefficients gave a total void worth of $58 \phi$ as compared to the measured value of $46.9 \pm 1.8 \phi$.

\section{Central Vacancy Lattice}

As shown in Figure 13, the voiding of the central seven vacant positions produced a significantly different pattem. Reactivity worth is observed to rise rapidly as the top third of the reactor is voided, level off in the middle third, and then rise rapidly again in the bottom third. The behavior is 


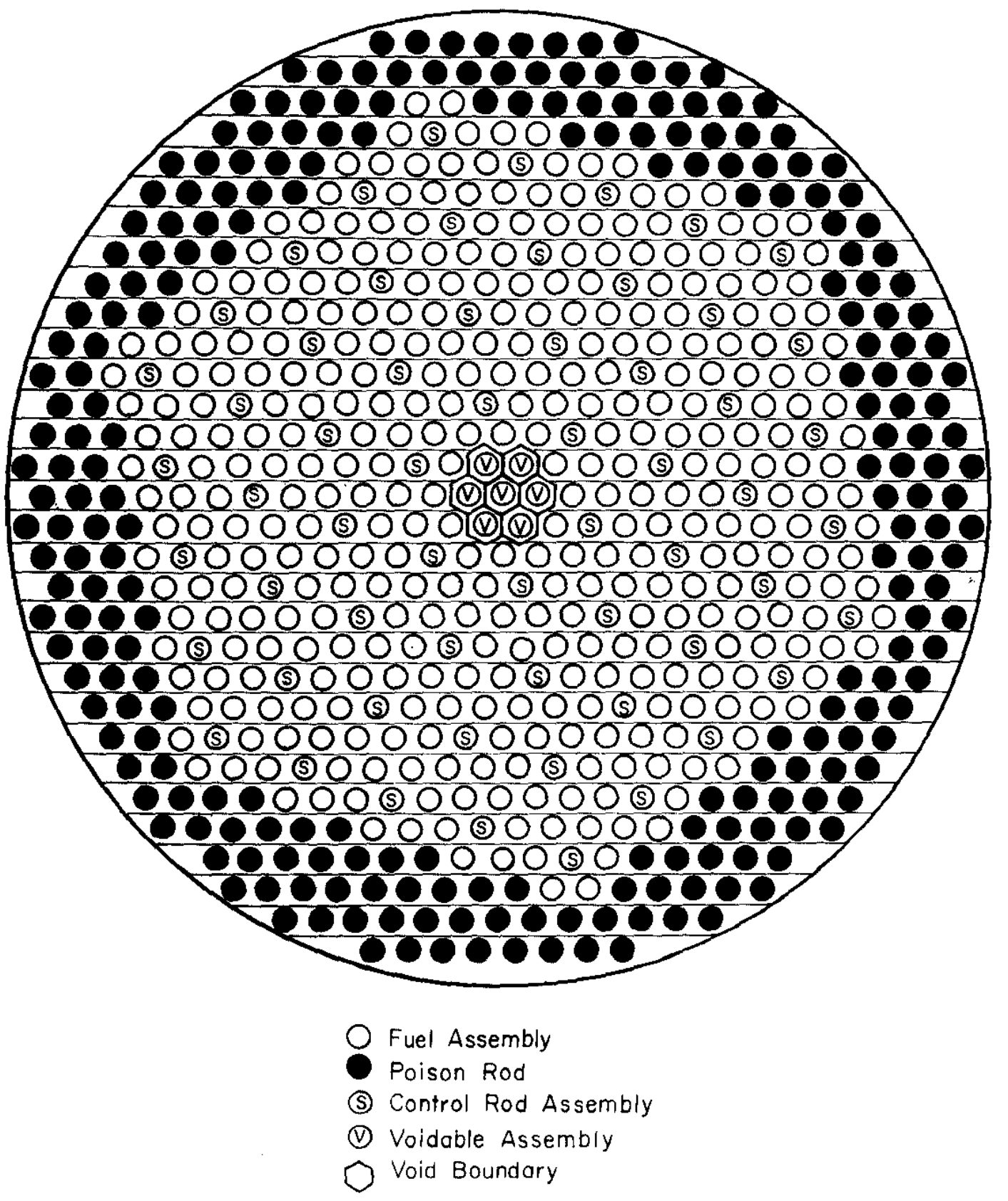

FIGURE 10. PDP Face Map for Enriched Lattice Loading 


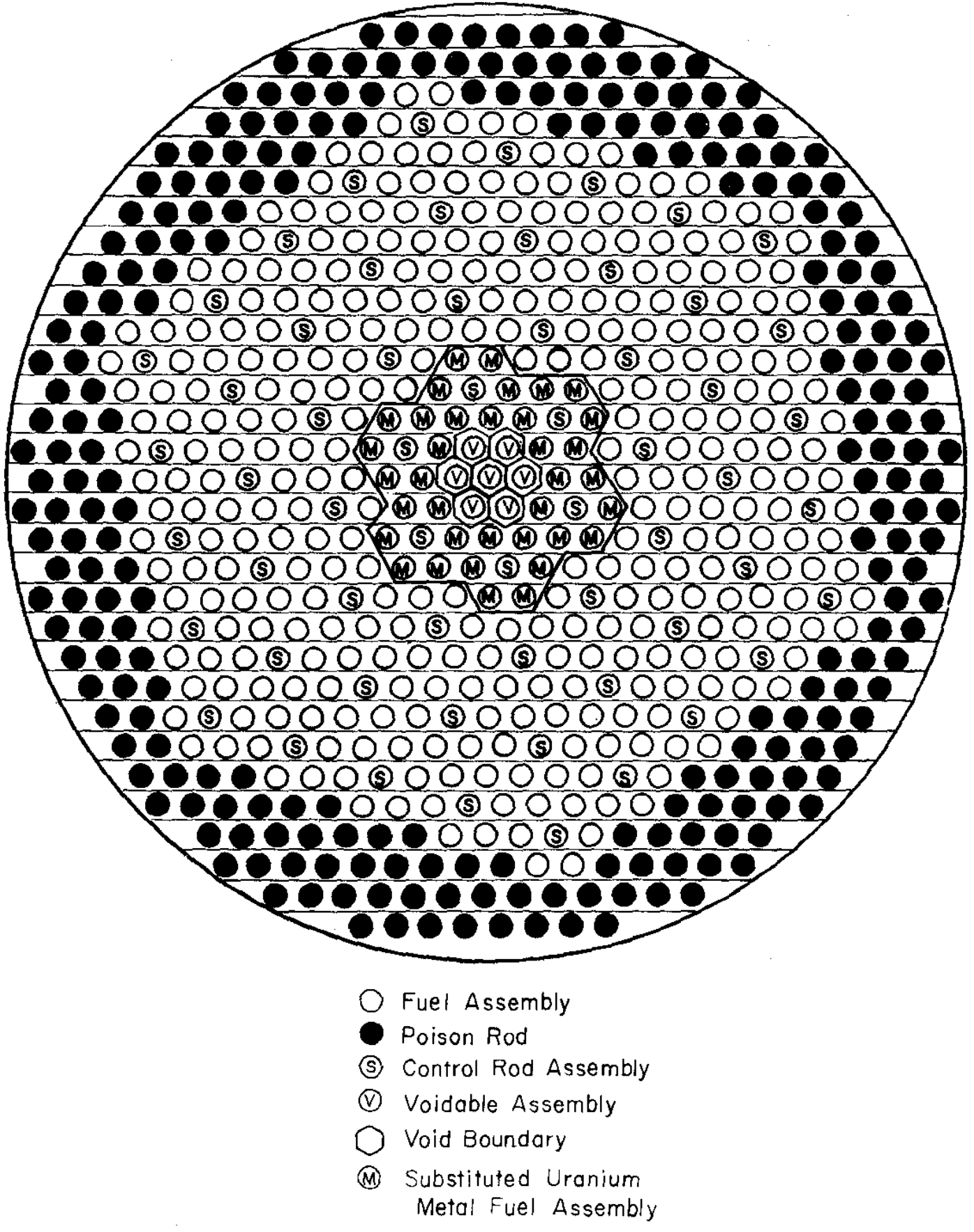

FIGURE 11. PDP Face Map for Uranium Metal Lattice by Substitution in Enriched Host Lattice 


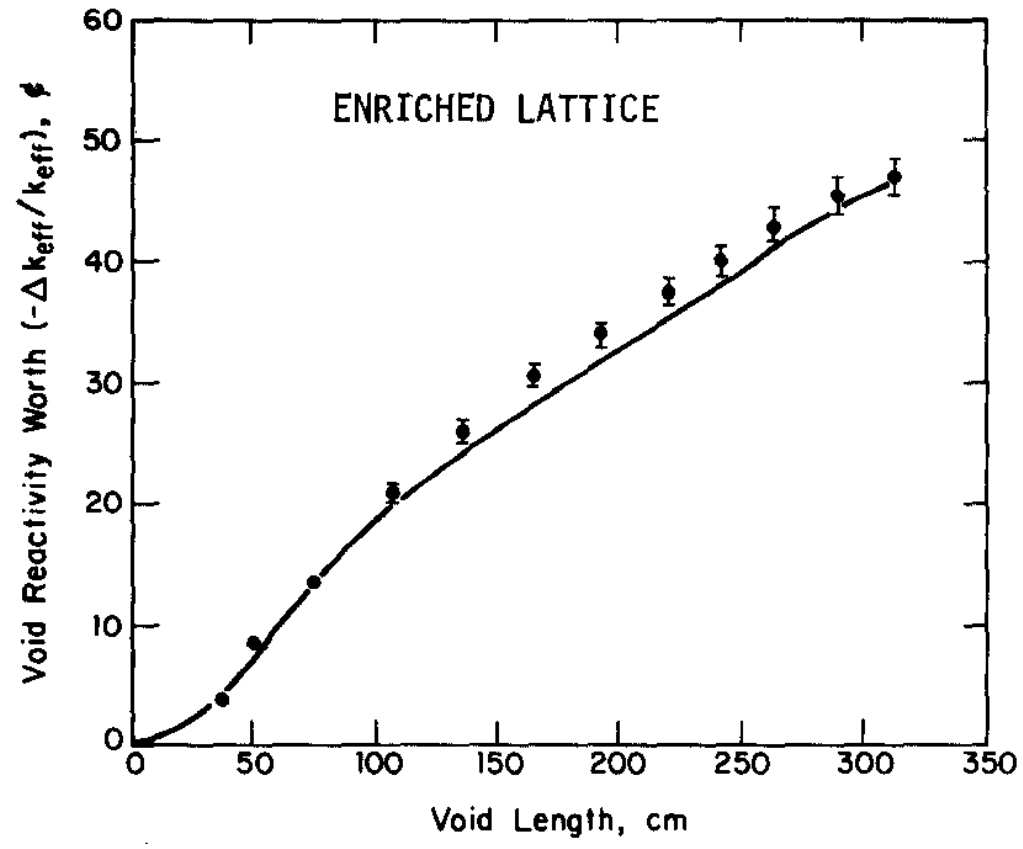

FIGURE 12. Comparison of Measured and Computed Reactivity Change as a Function of Void Length - Enriched Lattice 


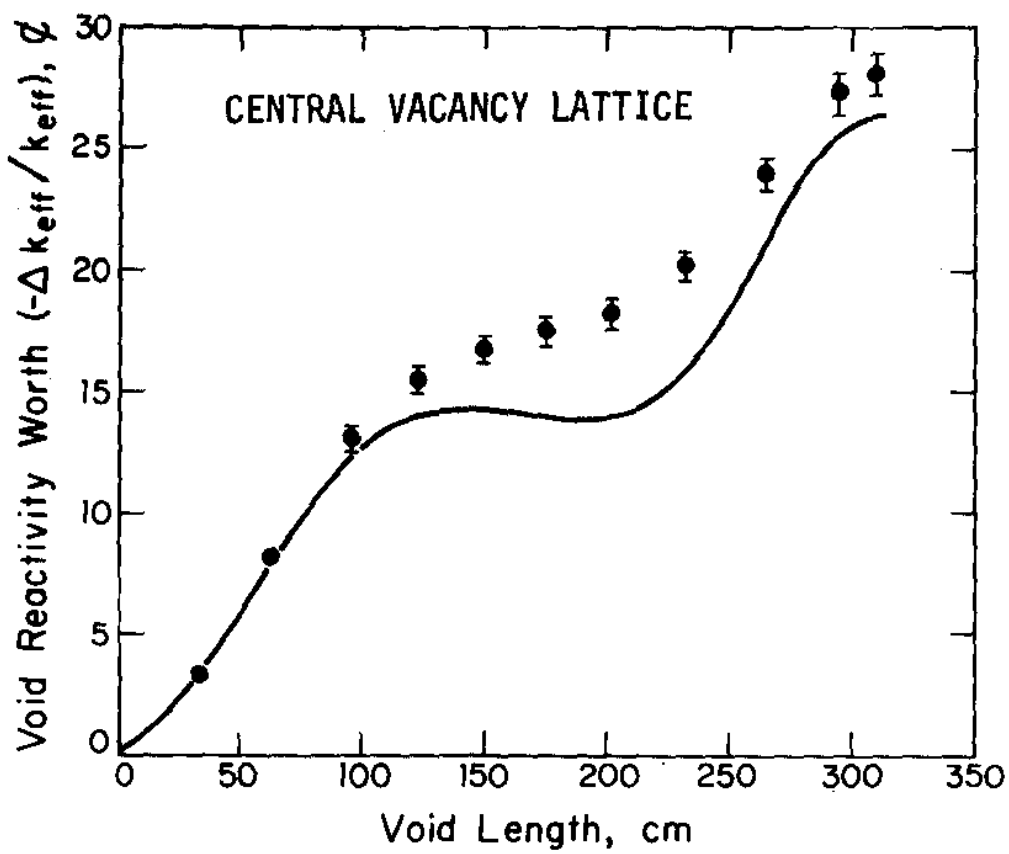

FIGURE 13. Comparison of Measured and Computed Reactivity Change as a Function of Void Length - Central Vacancy Lattice 
entirely consistent with the experimental design concept of a high leakage region with negligible neutron production and parasitic absorption. In this design, the rate of leakage increase is at a minimum at the reactor midplane, which is what the data is implying. The GRIMHX 3-D calculation (solid curve) apparently underestimates this leakage effect relative to cross section changes, but agrees very well near the top and bottom of the reactor. Possible explanations for the middle region discrepancy include small errors in input data for residual materials in the void region (primarily void cans) or, more likely, inadequacies in the model used to generate the diffusion parameters. No attempts were made to resolve this relatively small discrepancy ( 2 to $5 \phi$ ). A computed total void worth of $37.4 \$$ was obtained with GRIMHX 2D calculations versus a measured worth of $29.2 \pm 1.2 \$$.

\section{Uranium Metal Lattice}

The final worth measurements were made for the substituted Uranium Metal Lattice and are shown in Figure 14. Again good agreement is observed with the calculation slightly underpredicting the worth over the bottom two-thirds of the core. In all cases, the disagreement between experiment and calculation is greatest near the midplane of the reactor. A slight bias may exist in the data for this region since the migration area (see below) was measured only at the end points (base case and fully voided) with linear interpolation of all intermediate voids. Further remedial effort is probably not warranted, but any future void measurements should include migration area measurements at the midplane void configuration to aid in interpolation. 2-D calculations gave a full void worth of $42.1 \$$ compared to the measured value of $37.2 \pm 1.5 \$$.

In order to summarize and better compare all the void worth measurements, Table 3 was constructed with a step-by-step correction process. This better identified the direction and magnitude of the worth corrections due to spectrum (37-cel1 GLASS) and diffusion coefficient effects (LEAK). Note that the uncorrected GLASS cell calculations greatly overpredict $\Delta \mathrm{k}$ and that the spectrum correction increases this disagreement. However, the diffusion coefficient correction is so large that it offsets the overprediction and spectrum correction almost exactly. Two more observations are significant. First, the spectrum correction is apparently only a weak function of lattice type, increasing $\Delta \mathrm{k} 8$ to $10 \%$ in each case, whereas the diffusion correction is a very strong function of lattice type or, more specifically, of void region content. 


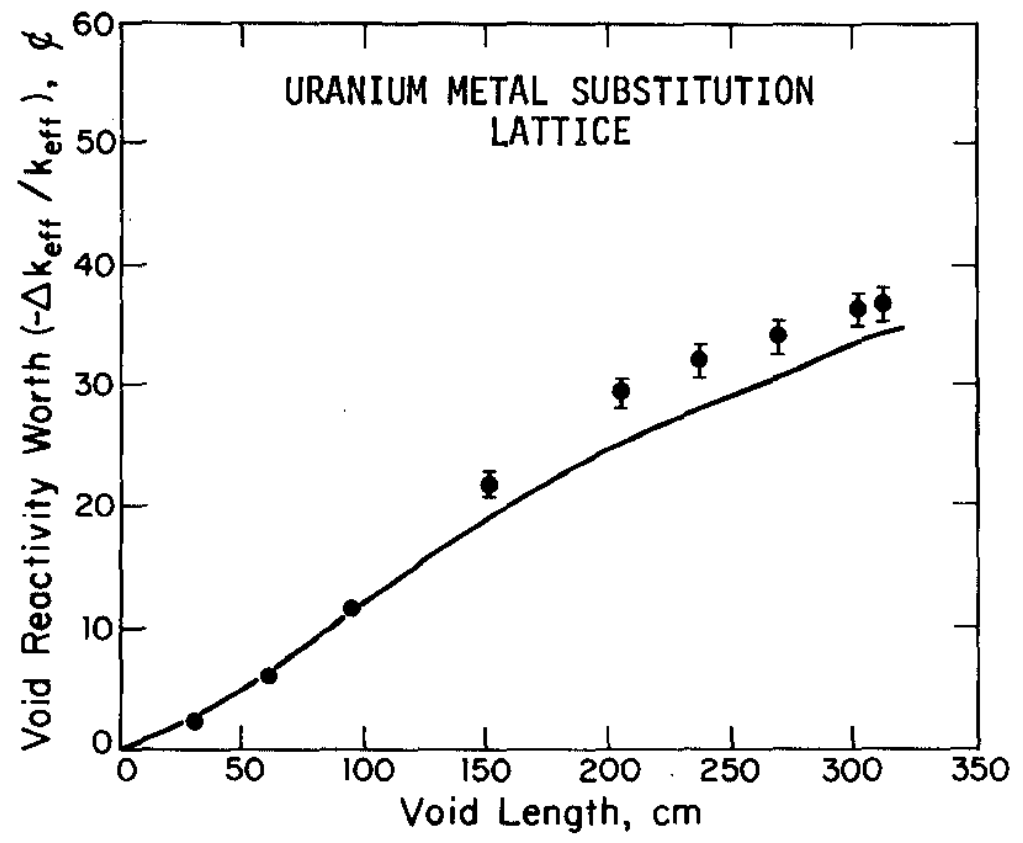

FIGURE 14. Comparison of Measured and Computed Reactivity Change as a Function of Void Length - Uranium Metal Lattice 
TABLE 3

Detailed Comparison of Measured and Calculated Void Worths*

\begin{tabular}{|c|c|c|c|c|c|}
\hline \multirow[b]{2}{*}{ Lattice Type } & \multirow[b]{2}{*}{$\begin{array}{c}\text { PDP } \\
\text { Measurement }\end{array}$} & \multicolumn{3}{|c|}{ GRIMEX 2-D } & \multirow[b]{2}{*}{$\begin{array}{l}G R I M H X^{* *} \\
3-D\end{array}$} \\
\hline & & $\begin{array}{l}\text { Single-cell } \\
\text { GLASS }\end{array}$ & $\begin{array}{l}37-c e 21 \\
\text { GLASS }\end{array}$ & $\begin{array}{l}37-c e Z 2 \\
G L A S S+L E A K\end{array}$ & \\
\hline Enriched Lattice & $46.9 \pm 1.8$ & 77.1 & 84.8 & 58.0 & 47.1 \\
\hline $\begin{array}{l}\text { Central Vacancy } \\
\text { Lattice }\end{array}$ & $29.2 \pm 1.2$ & 78.7 & 86.4 & 37.4 & 26.5 \\
\hline Natural Uranium & $37.2 \pm 1.5$ & 54.1 & 58.3 & 42.1 & 34.2 \\
\hline
\end{tabular}

* All reactivity worths in $(\alpha)\left(B_{\text {eff }}=0.0072\right)$.

** Using 37-cell GLASS + LEAK. 


\section{Migration Areas}

Migration areas for each of the test lattices are shown in Table 4. The probable errors quoted include uncertainties in critical $\mathrm{D}_{2} \mathrm{O}$ height, counting statistics, and, by far the predominant contributor, uncertainties in the $\beta_{i}$ 's (delayed neutron family components). The direct delayed neutron data $\left(\beta_{i_{0}}\right.$ and $\left.\lambda_{i}\right)$ are taken from the compilation and evaluation of Tuttle, ${ }^{8}$ while the $\mathrm{D}_{2} \mathrm{O}$ photo-delayed data is from a compilation by Church. ${ }^{9}$ The values of $\beta_{\text {eff }}$ entered in the table are computed by the point reactor kinetics code. These $\beta_{e f f}$ values are used in expressing void worths in Beff units (i.e., dollars and cents). It should be noted that differences in the $\mathrm{M}^{2}$ from base to fully voided cases are sma11 (5 to $7 \%$ ), and generally less than the uncertainties in the data. Differences due to lattice type are almost negligible.

\section{Flux Shape Data}

\section{Radial Flux}

As stated earlier, radial flux data were obtained from both bare and Cd-covered gold pins which were irradiated in each lattice configuration investigated and subsequently counted on an automatic sample changer system. These data were used to evaluate the reactor calculations and determine if any appreciable discrepancies were present. None of the lattice configurations tested showed large discrepancies except near and in the void region. Spectrum changes are significant in this region, and special corrections for the data were required. Correction of these few data points for spectral effects was not done since a complete spectral investigation (see later section) of the void region was performed to assist with modifications to the GLASS cell calculations. In the subsequent Figures 15-20, the measured void-region flux data point is not plotted, but the GRIMHX calculated flux is plotted to zero radius. The GRIMHX calculated flux shape is normalized to the experimental data at approximately 90 $\mathrm{cm}$ radius. Note that the radial flux shape is greatly perturbed by the presence of a central void. Calculated flux shapes over the unvoided portion of the reactor must be in reasonably good agreement before spectrum and diffusion coefficient corrections are made. Uncertainties in radial flux data are not easy to define quantitatively. The statistical counting errors are generally less than $1 \%$, but positioning of the pins primarily in the radial direction may lead to 5 to $10 \%$ errors or more. Variation in gold pin weights and purity are responsible for less than $1 \%$ uncertainty. With the agreement between calculation and experiment observed by radial fluxes in the unperturbed regions of the reactor, it was concluded that the basic reactor calculation was satisfactory and that additional work would concentrate on cell parameter generation or in axial flux discrepancies.

$$
\text { - } 39 \text { - }
$$




\section{TABLE 4}

Measured Migration Areas for Moderator Void Test Lattices

Lattice Type

Enriched Lattice

Central Vacancy Lattice

Natural Uranium Metal Lattice

$\frac{M^{2}\left(\mathrm{~cm}^{2}\right)}{\text { Base Case Full Void }}$

$177 \pm 9$

$189 \pm 9$

$172 \pm 9$

$180 \pm 9$

$177 \pm 9$

$185 \pm 9$ $\beta_{e f f}$

$0.00718 \pm 0.00036$

$0.00718 \pm 0.00036$

$0.00735 \pm 0.00037$ 


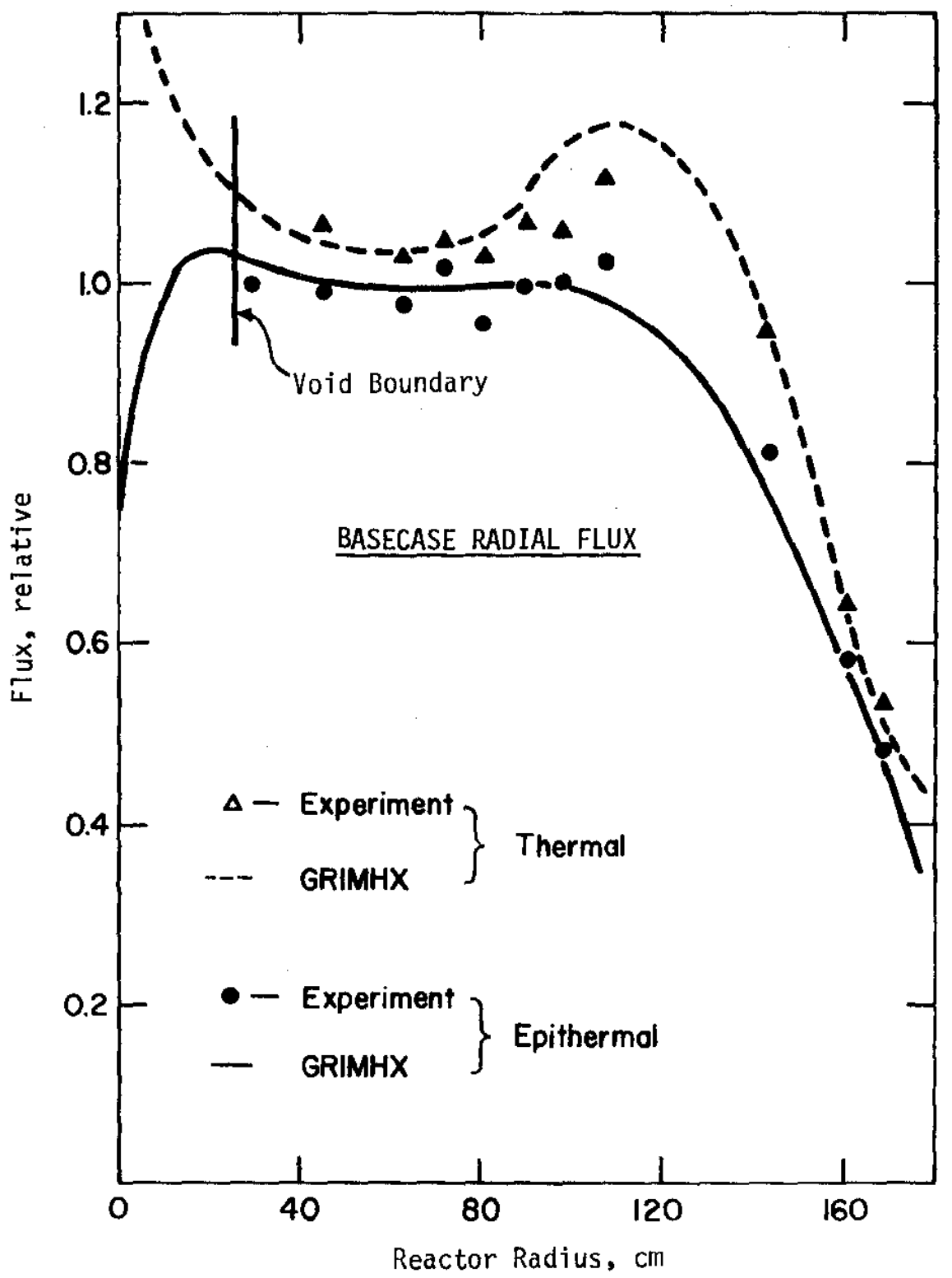

FIGURE 15. Comparison of Measured and Computed Radial Flux Shapes - Enriched Lattice - Base Case 


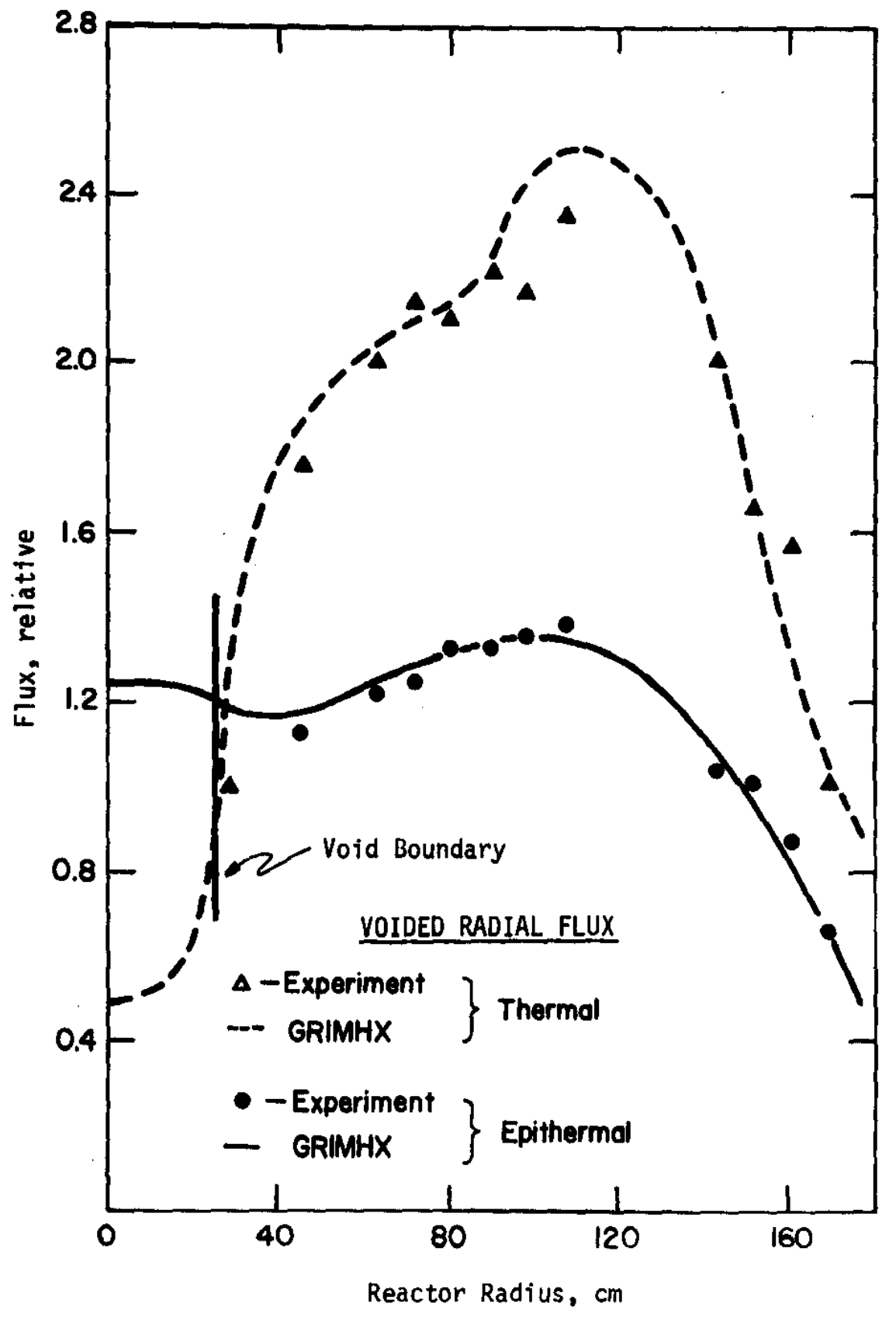

FIGURE 16. Comparison of Measured and Computed Radial Flux Shapes - Enriched Lattice - Voided 


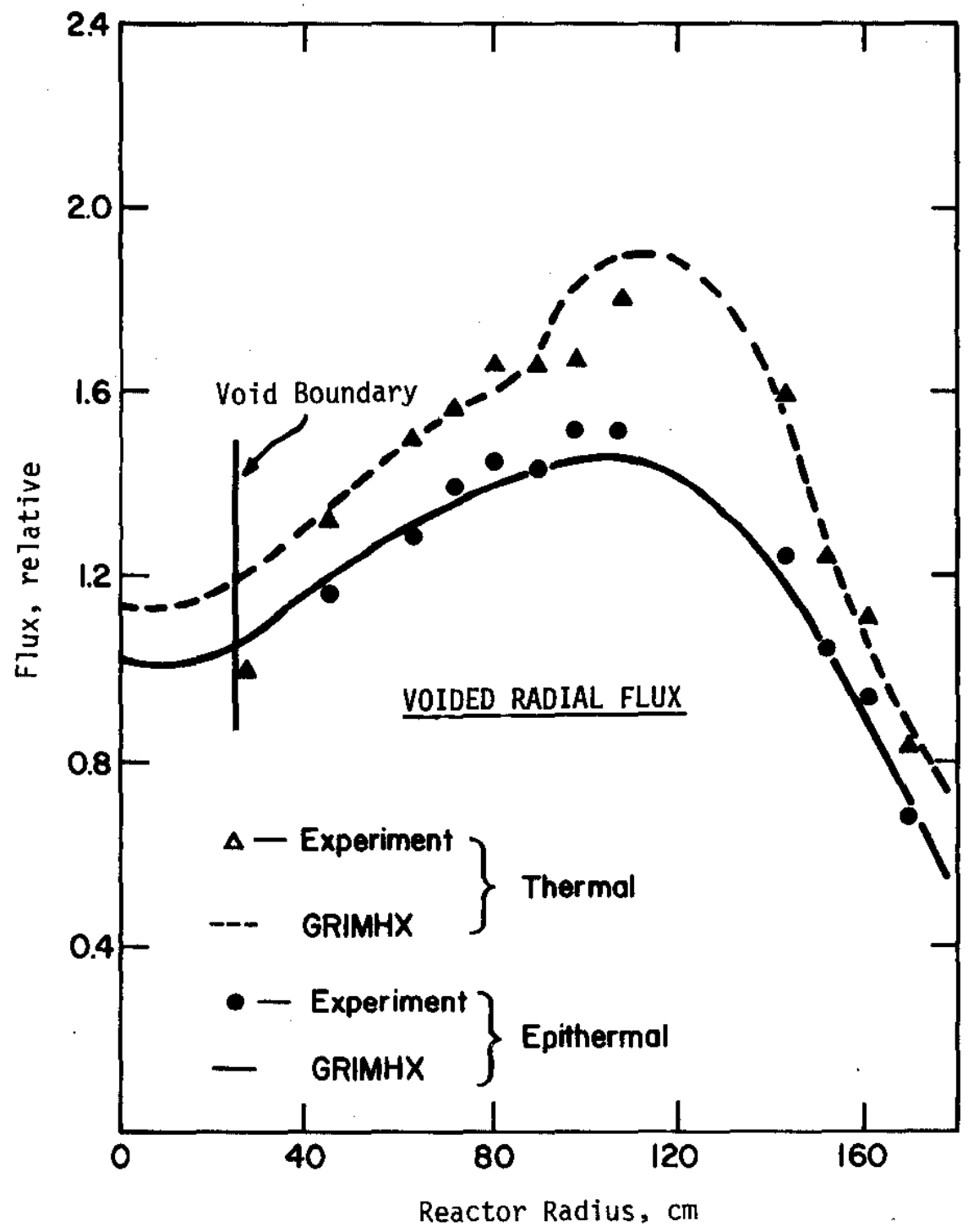

FIGURE 17. Comparison of Measured and Computed Radial Flux Shapes - Central Vacancy Lattice - Base Case 


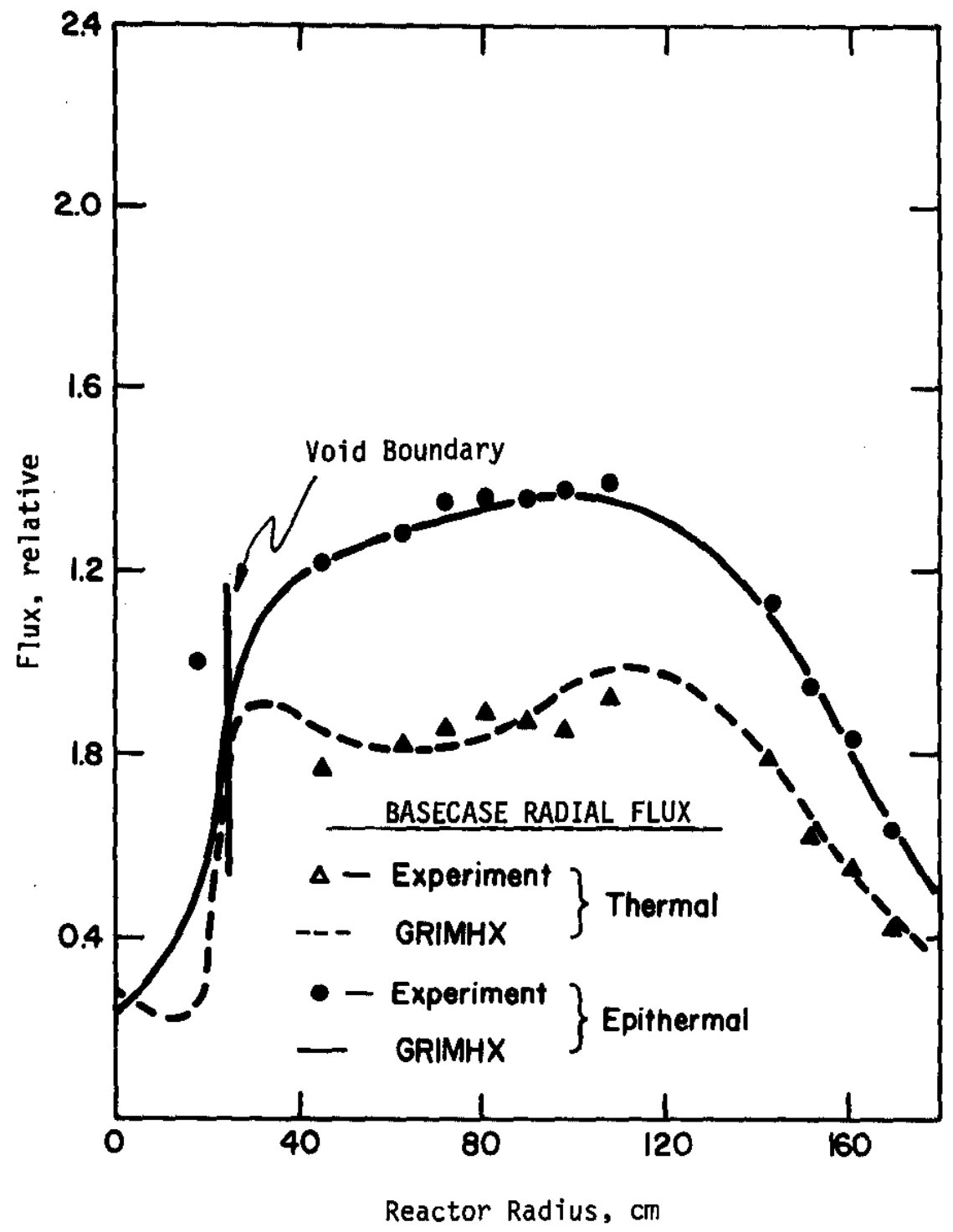

FIGURE 18. Comparison of Measured and Computed Radial Flux Shapes - Central Vacancy Lattice - Voided 


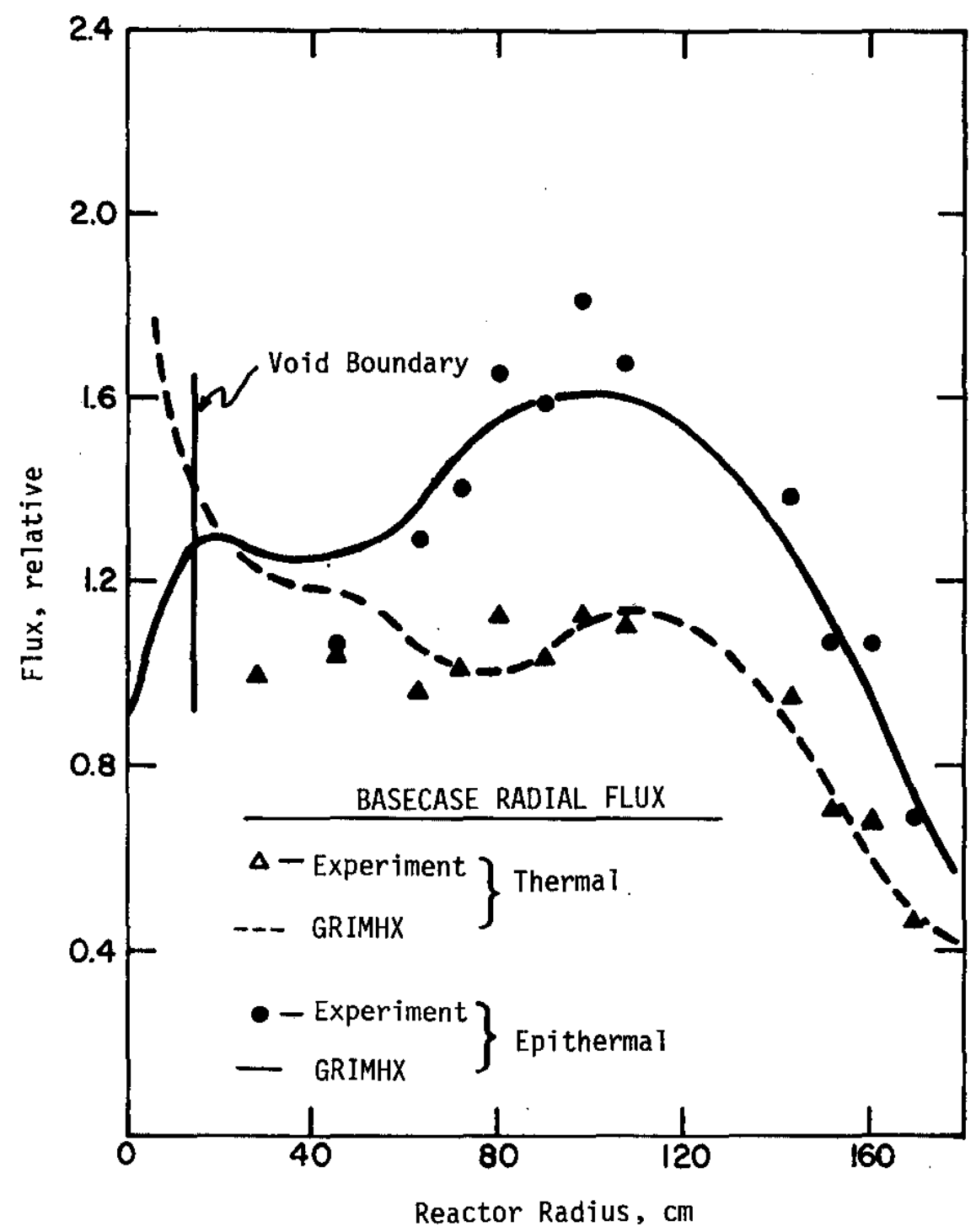

FIGURE 19. Comparison of Measured and Computed Radial Flux Shapes - Uranium Metal Lattice - Base Case 


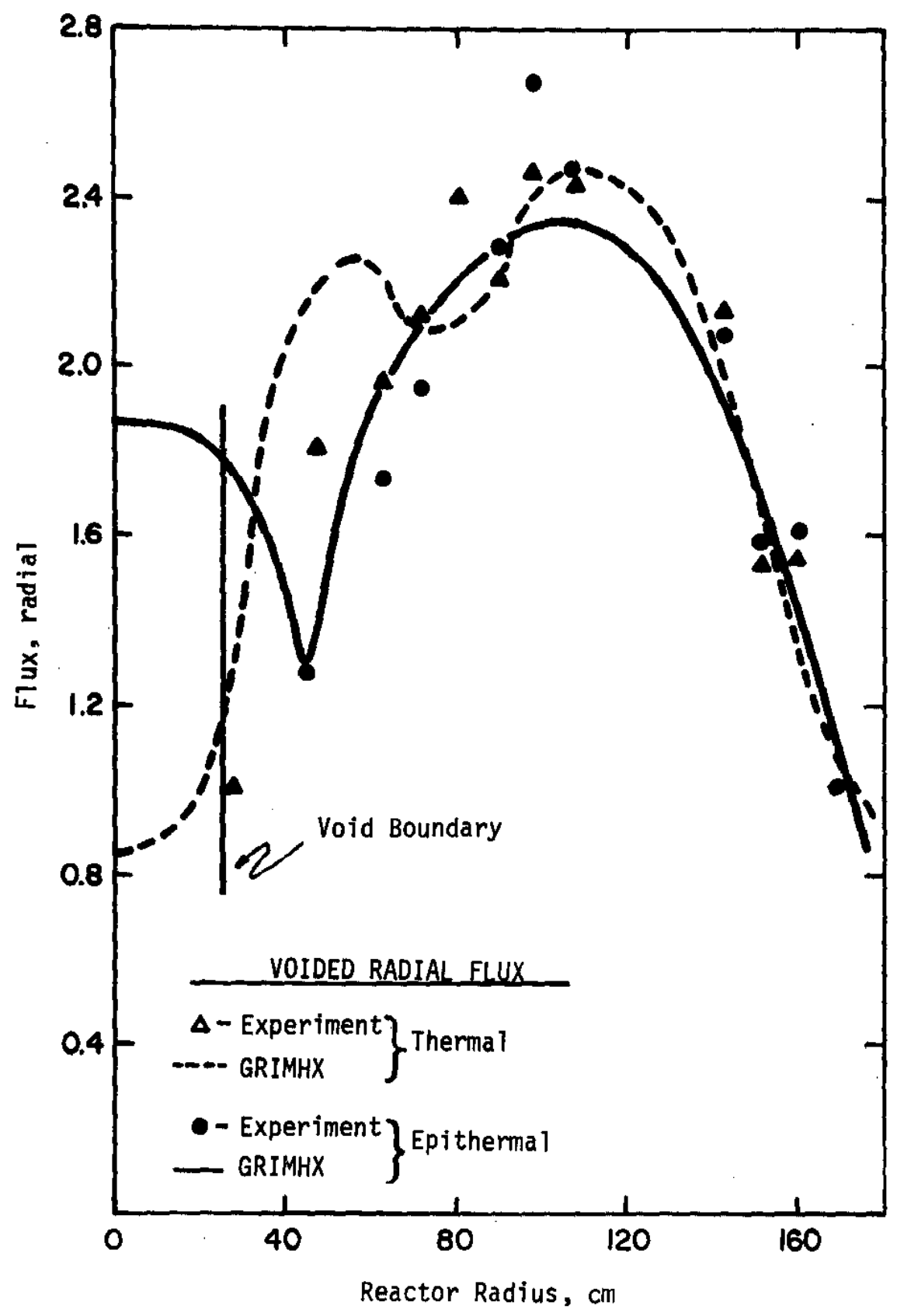

FIGURE 20. Comparison of Measured and Computed Radial Flux Shapes - Uranium Metal Lattice - Voided 


\section{Axial Flux}

Axial flux shapes are generally measured in all reactor experiments to obtain the top and bottom extrapolation distances for inclusion in 2-D reactor calculations. For 3-D calculations, the axial flux shapes provide information to test the ability of the code to model the axial geometric detail of the reactor. A brief description of axial flux discrepancies was given in the GRIMHX-3D theoretical section earlier in this report. Basically, the 3-D calculation does not give the proper axial flux shape if the model is terminated at the critical $\mathrm{D}_{2} \mathrm{O}$ height (Figure 8). This discrepancy results from the fact that leakage neutrons interact with the fue 1 extending above the $\mathrm{D}_{2} \mathrm{O}$ and raise the "effective" top boundary of the reactor. This effect is extremely important to the worth of the first 20 to $50 \mathrm{~cm}$ of voiding, and introduces a non-negligible error for all void configurations. The remedy was simply to expand the top boundary of the GRIMHX core to the physical top of the PDP fuel and to insert voided fewgroup parameters for this extended top region (Figure 9). The method yielded excellent agreement between calculated and experimenta1 axial fluxes (Figures $8,21,22$ ), but more important, this change in reactor geometry afforded greatly improved $\Delta \mathrm{k}$ calculations as the top third of the void region was voided.

Whether the calculation is for void effects or otherwise, the top boundary region should be included in all future PDP 3-D calculations whenever possible. Critical $\mathrm{D}_{2} \mathrm{O}$ height predictions should be improved by 5 to $15 \mathrm{~cm}$ with inclusion of the extended fuel region. Rod worth and space-time measurements also require this top boundary method for accurate computed results.

Voided axia1 flux shapes are much more complex to analyze and are a strong function of void configuration. Figure 23 shows three thermal axial flux profiles for the enriched lattice. The axial fluxes were measured at the radial center of the void region for the base case and for voids of 136.5 and $262.6 \mathrm{~cm}$. The data points are indicated by solid circles and the normalized GRIMHX fluxes by the solid curve. Thirty-seven axial mesh points were used in GRIMHX to obtain these results. Figure 24 shows the corresponding epithermal fluxes for the same reactor configurations. However, the combination of strong spectral changes and the $1 / \mathrm{v}$ nature of the detector preclude a direct comparison of experiment and calculation. The solid circles represent the raw data which are actually the boron reaction-rate in the detector. The solid curves are the GRIMHX Group 1 (2-group treatment) flux calculations normalized to the data at the peak values. Note that at the void interface, the flux behaves in a very different manner from the reaction-rate data. This results from changes in the resonance integral due to spectrum changes. At present GRIMHX does not have 


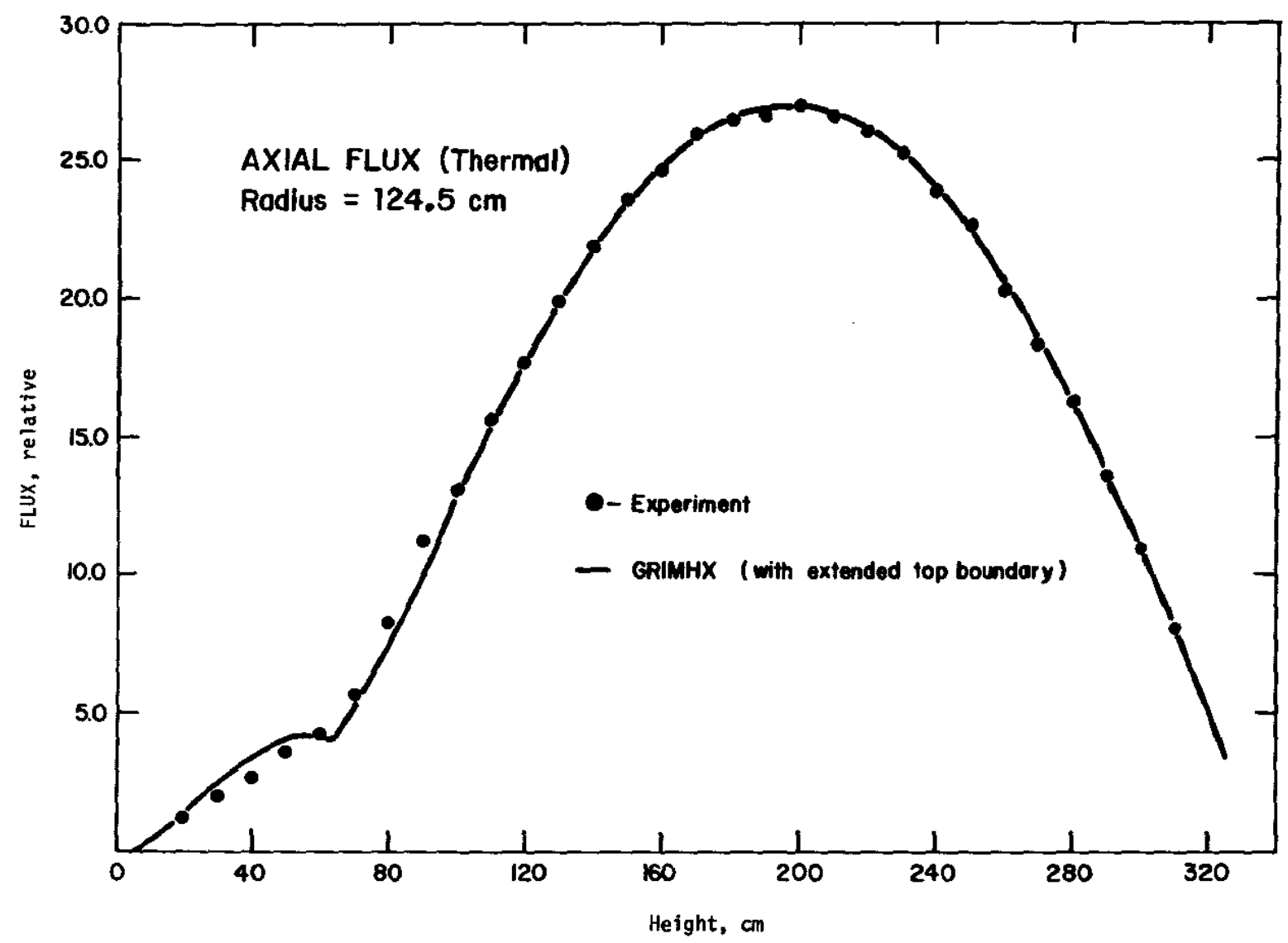

FIGURE 21. Thermal Axial Flux Shape Measured and Calculated with GRIMHX Central Vacancy Lattice 


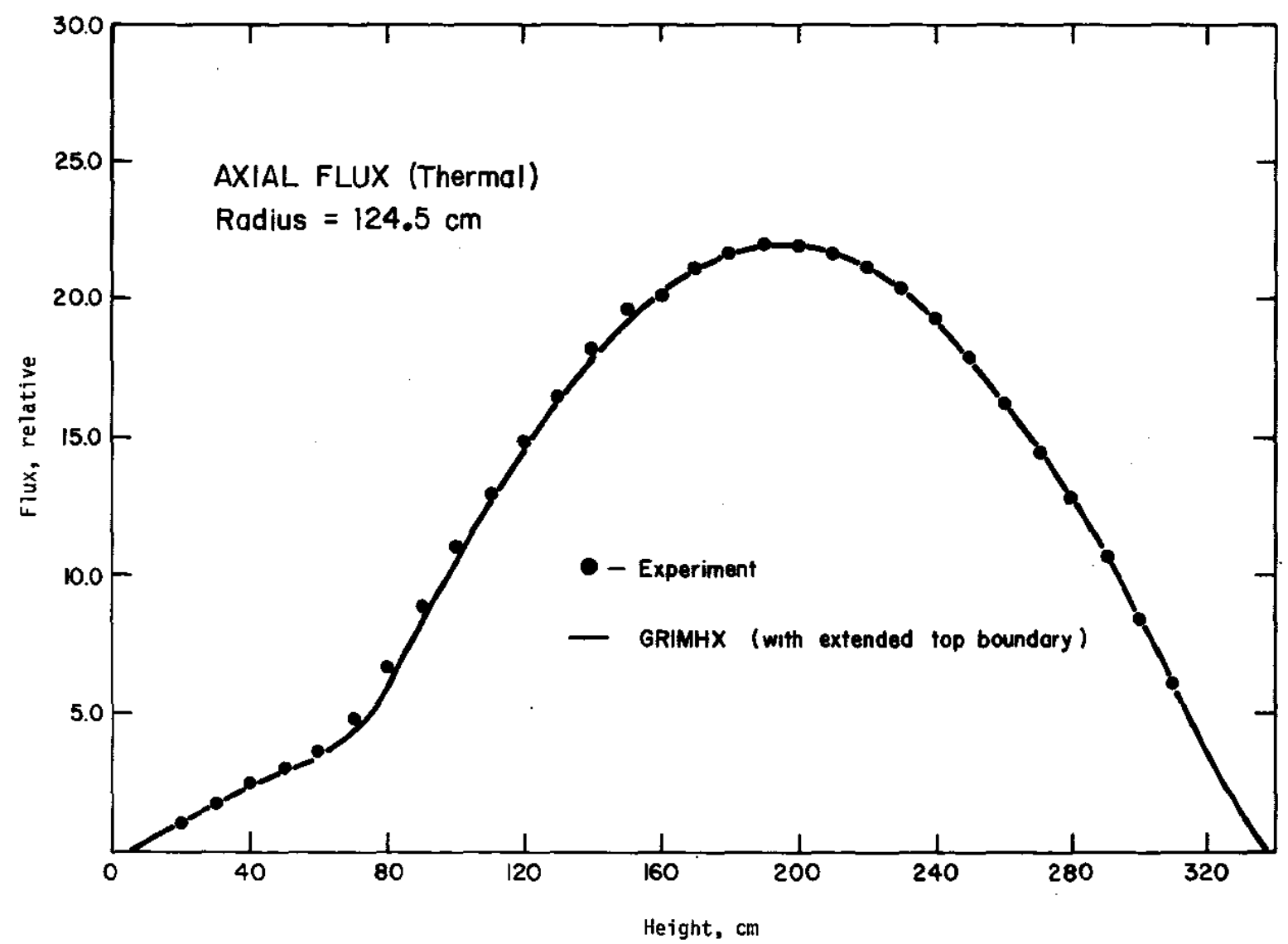

FIGURE 22. Thermal Axial Flux Shape Measured and Calculated with GRIMHX Uranium Metal Lattice 


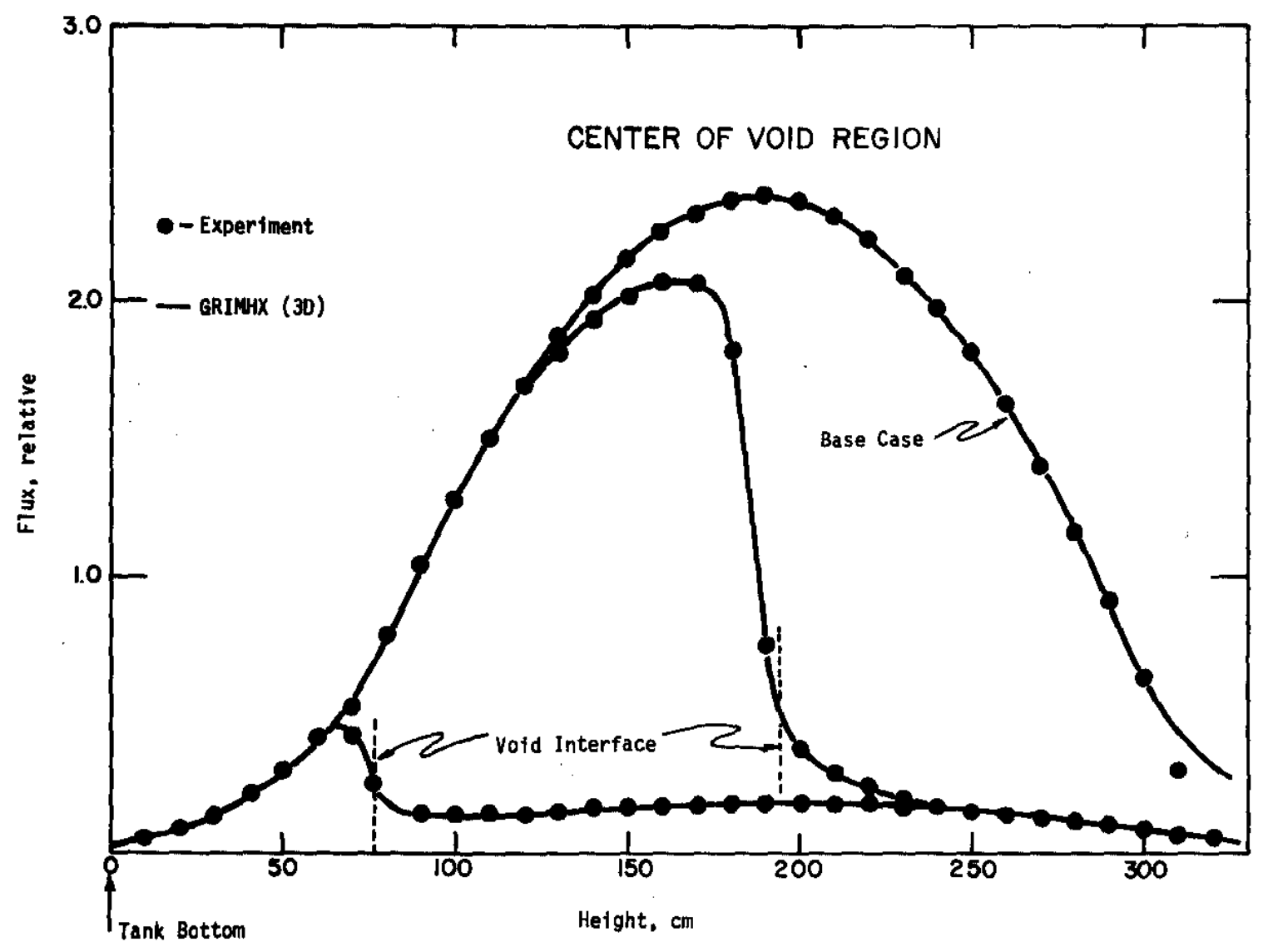

FIGURE 23. Comparison of Experimental and Theoretical Axial Flux Shapes for Moderated, Partial, and Fully Voided Central Void Region - Enriched Lattice - Thermal 


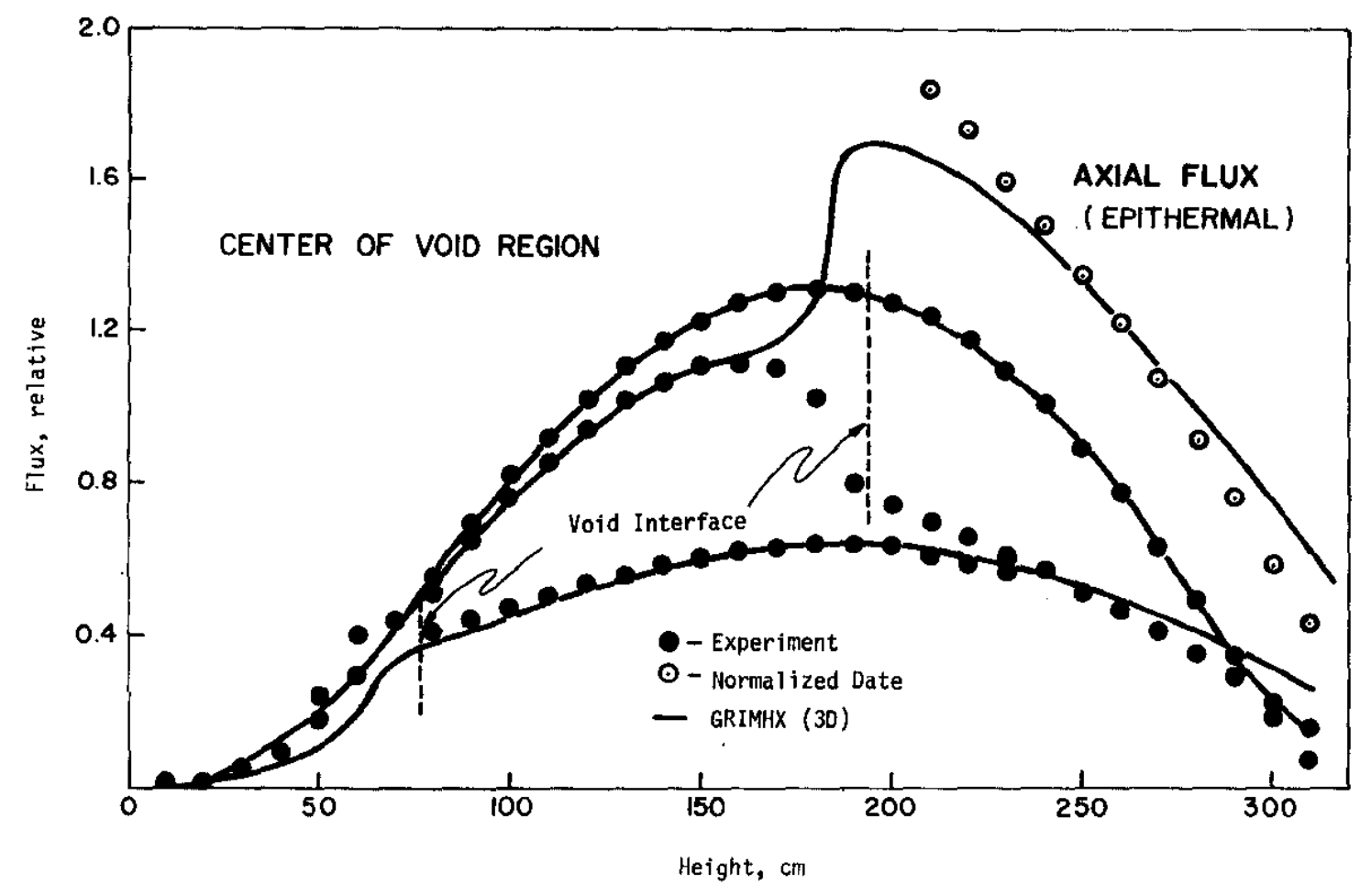

FIGURE 24. Comparison of Experimental and Theoretical Axial Flux Shapes for Moderated, Partial, and Fully Voided Central Void Region - Enriched Lattice - Epithermal 
a reaction-rate edit for axial traverses, but fission rates are edited. This fact suggests that future axial flux profiles should be measured with a ${ }^{2}{ }^{3} \mathrm{U}$ fission chamber rather than a boron-lined ion chamber in order to have direct comparison to calculation. For the present data, a uniform normalization of the data midway up the voided portion of the central patch has been performed and plotted with the open circles. Except for data points in the transition (interface) region, reasonable agreement is observed which implies that the epithermal flux calculation is probably consistent. Figures 25-28 are similar plots of the same type of data for the Central Vacancy and Uranium Metal Lattices.

\section{Axial Leakage FTux}

The axial leakage flux data were obtained in an attempt to experimentally modify isotropic infinite-lattice diffusion coefficients for the anisotropic effects of voiding. The fluxes plotted in Figures 29-31 are the actual leakage rates into a fixed solid angle representing only those neutrons leaving a constant surface area at the core boundary (Figure 3). Unfortunately, attempts to reproduce the data by LEAK calculations were unsuccessful. It is apparent that a much more sophisticated model is necessary to perform this type of calculation. The experimental data are of interest in a qualitative fashion in that leakage deviates from a pure cosine shape. If leakage were axially uniform (i.e., a cosine axial shape), a constant $D_{\text {axial }}$ would be appropriate. For the Enriched and Uranium Metal Lattices, an approximate cosine shape is observed. However, for the Central Vacancy Lattice, large deviations are observed from a cosine shape, adding evidence to the earlier speculations that a constant coefficient in the axial direction for this particular lattice may be aphysical. Note that in the leakage data, the solid angle was fixed such that normal changes in leakage due to geometry of the void are removed.

Additional data were obtained with a variable solid angle such that the entire void interface area was always observed by the detector/collimator. These data yielded no additional insight and were also not amenable to calculated comparisons.

\section{Neutron Energy Spectra}

\section{Enriched Lattice}

Neutron energy spectrum measurements by the resonance foil 1 technique were corrected for background and decay. Several additional corrections were then made. The first correction was made to the thermal reference data since a true thermal spectrum

$$
-52-
$$




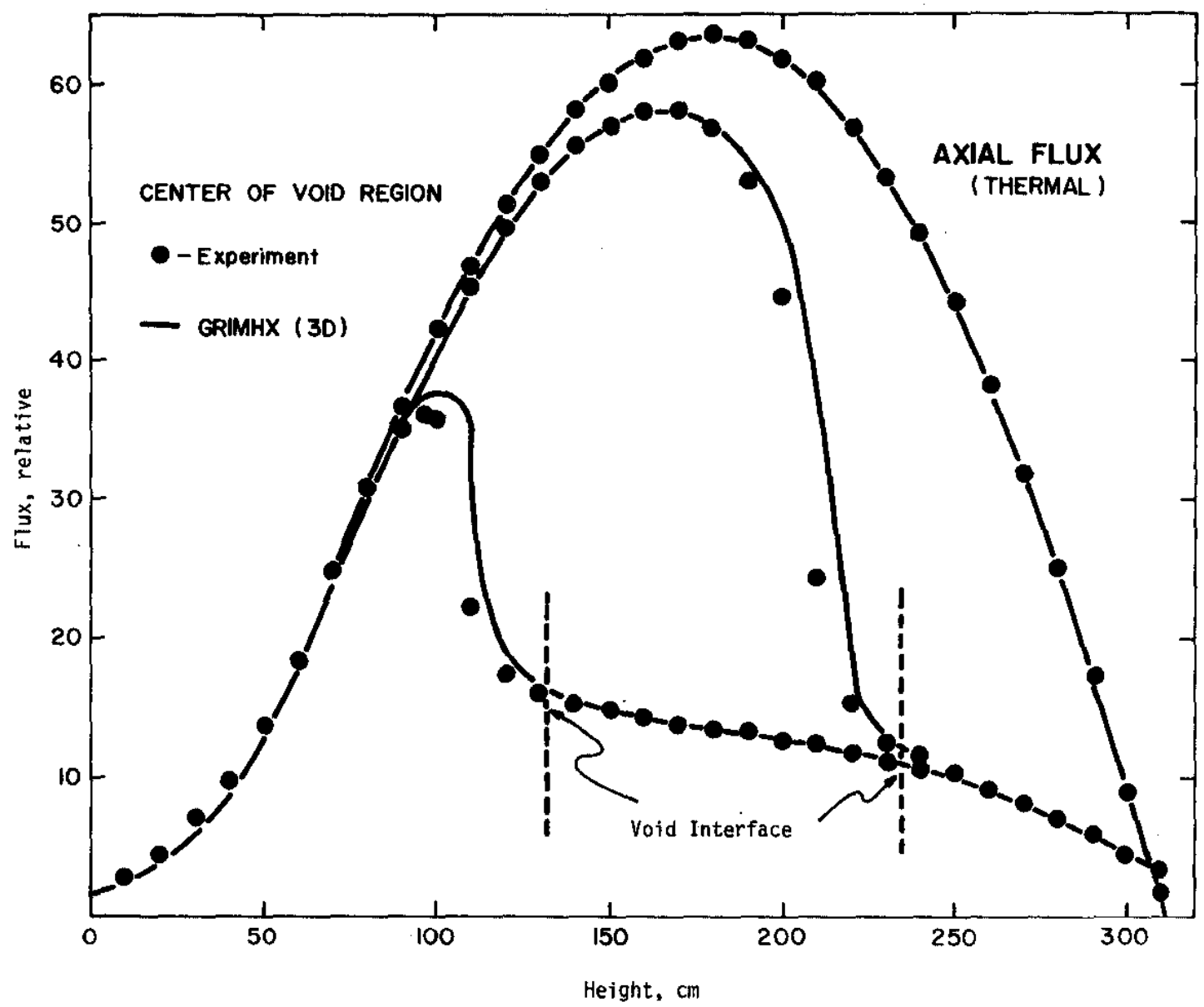

FIGURE 25. Comparison of Experimental and Theoretical Axial Flux Shapes for Moderated, Partial, and Fully Voided Central Void Region - Central Vacancy Lattice - Thermal 


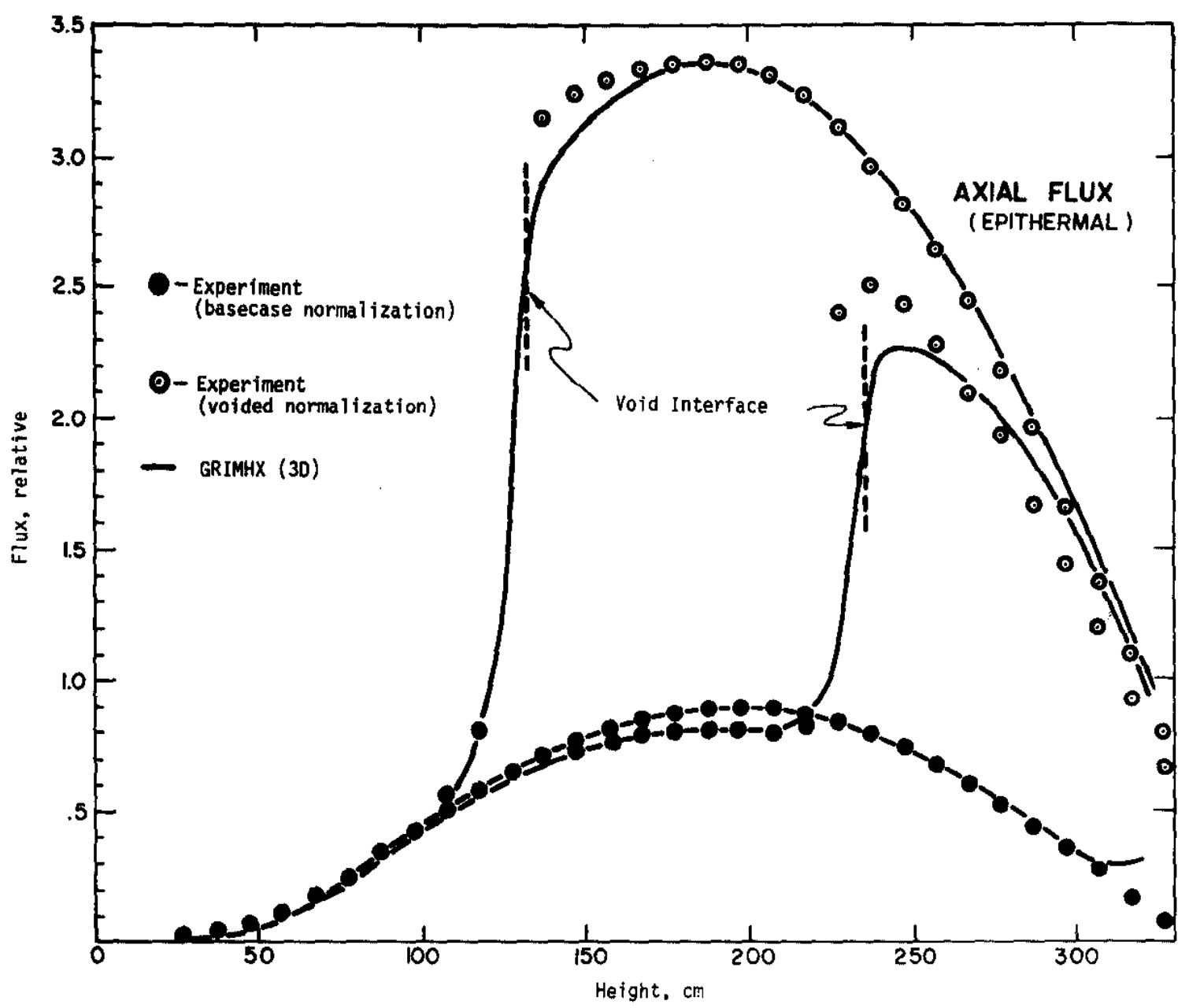

FIGURE 26. Comparison of Experimental and Theoretical Axial Flux Shapes for Moderated, Partial, and Fully Voided Central Void Region - Central Vacancy Lattice - Epithermal 


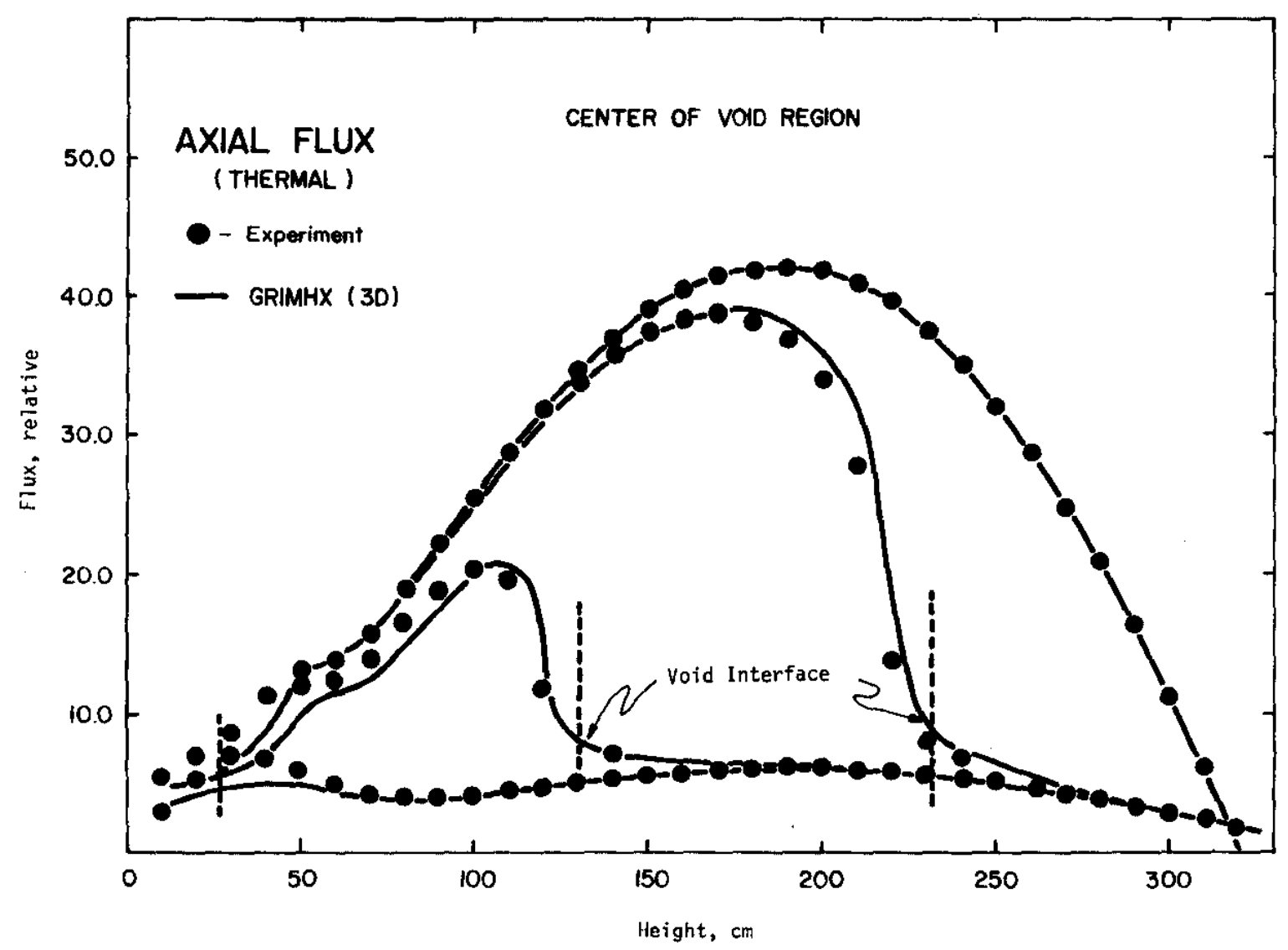

FIGURE 27. Comparison of Experimental and Theoretical Axial Flux Shapes for Moderated, Partial, and Fully Voided Central Void Region - Uranium Metal Lattice - Thermal 


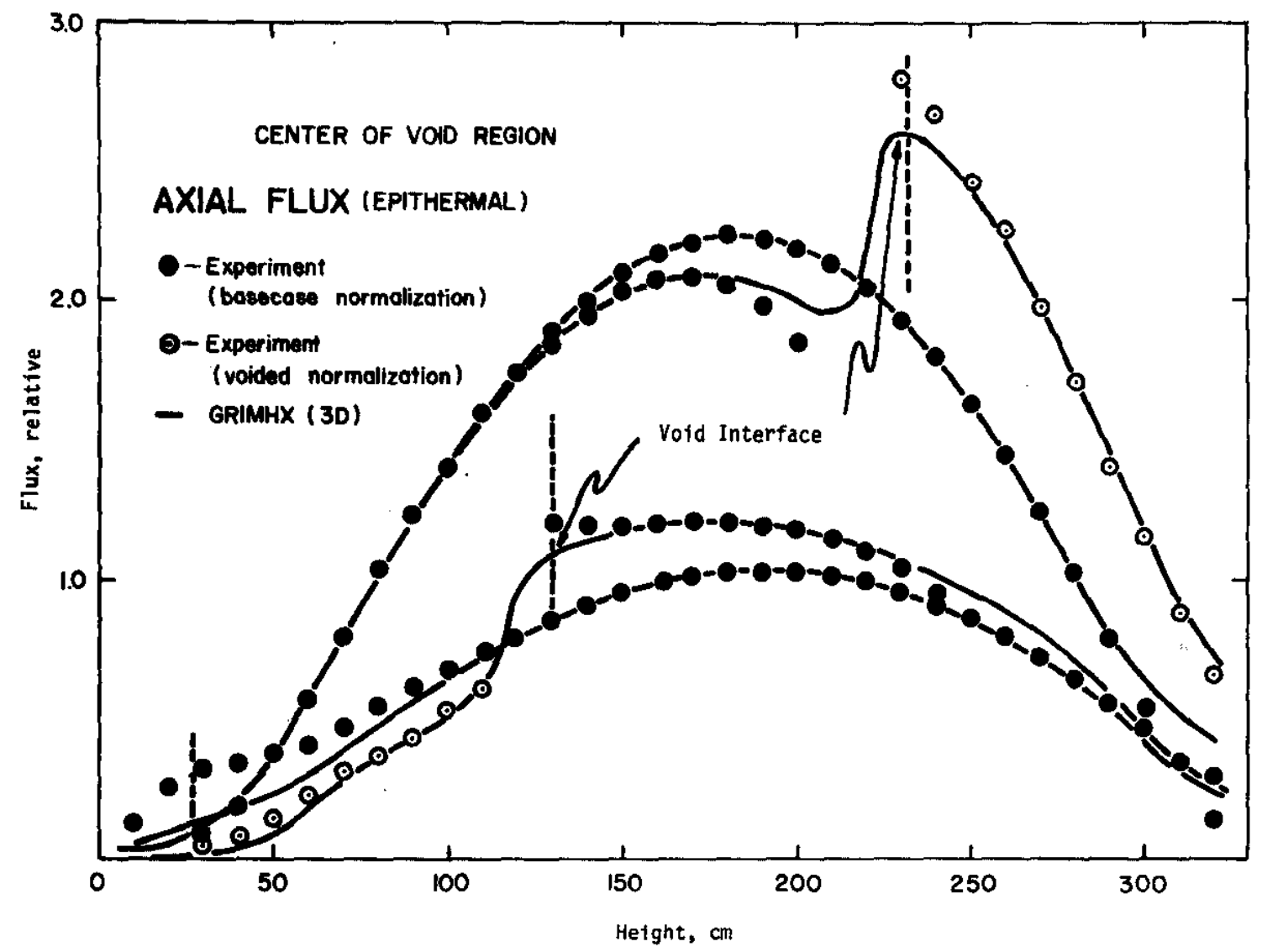

FIGURE 28. Comparison of Experimental and Theoretical Axial Flux Shapes for Moderated, Partial, and Fully Voided Central Void Region - Uranium Metal Lattice - Epithermal 


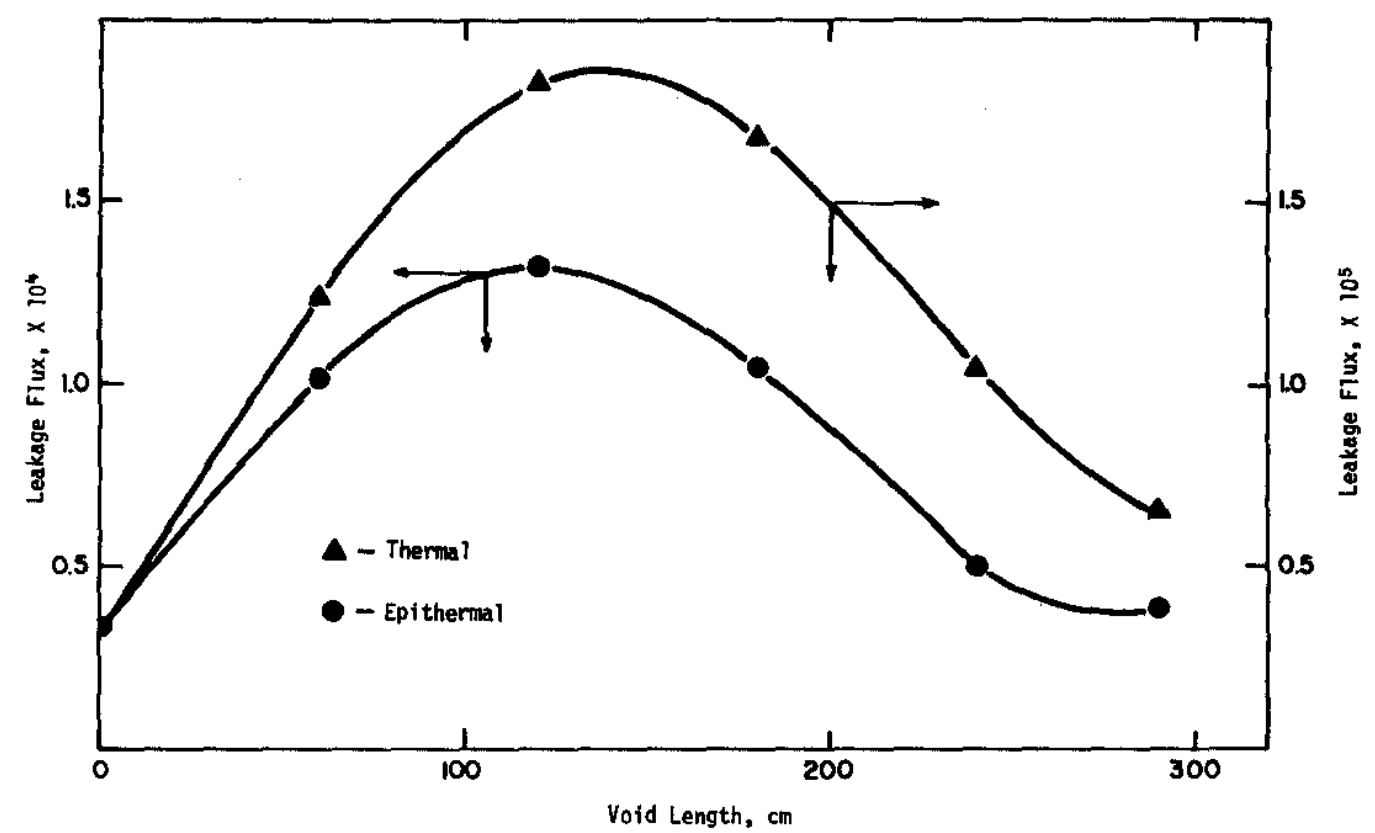

FIGURE 29. Measured Leakage Flux Data as a Function of Void Length - Enriched Lattice 


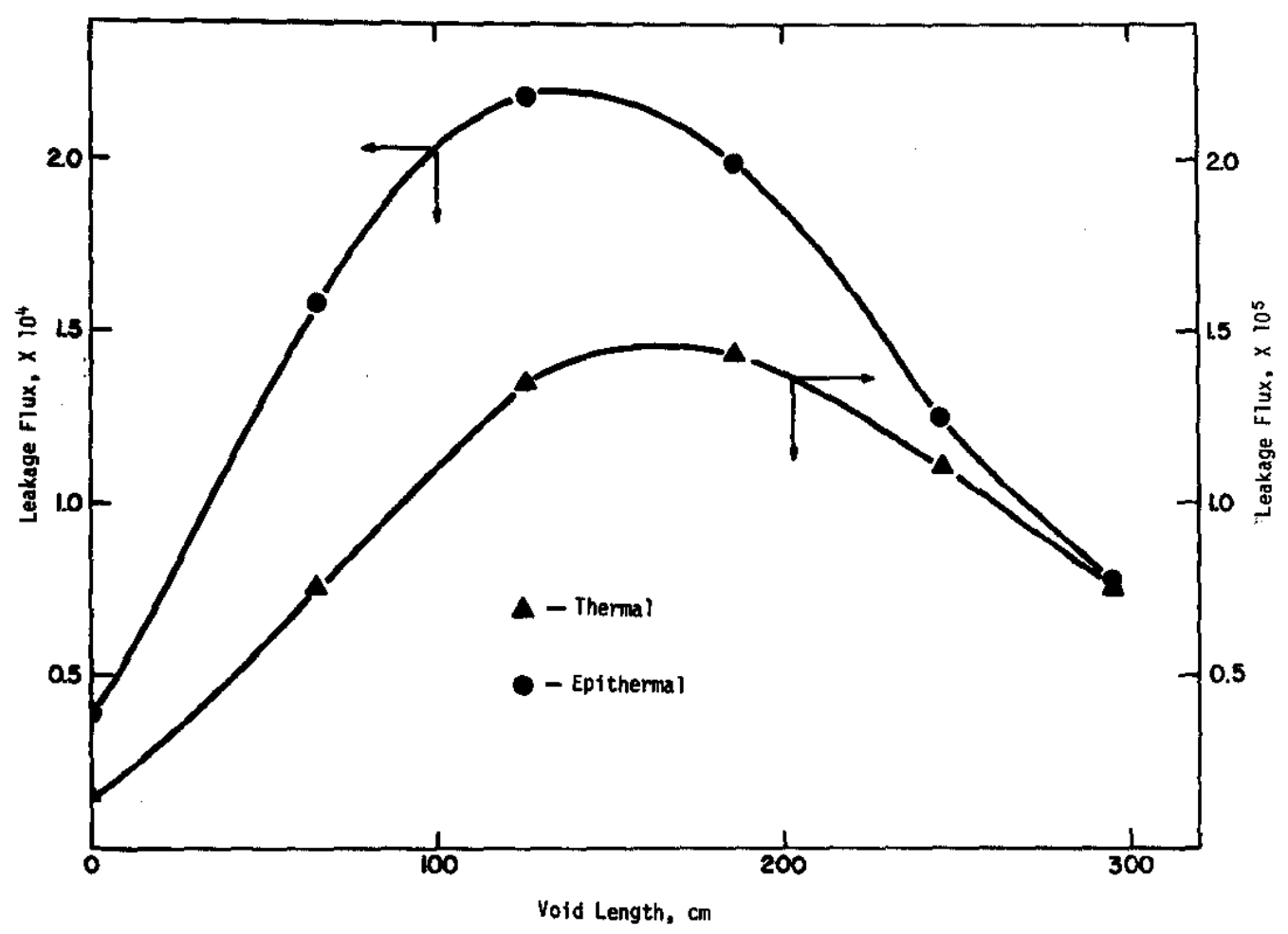

FIGURE 30. Measured Leakage Flux Data as a Function of Void Length - Central Vacancy Lattice 


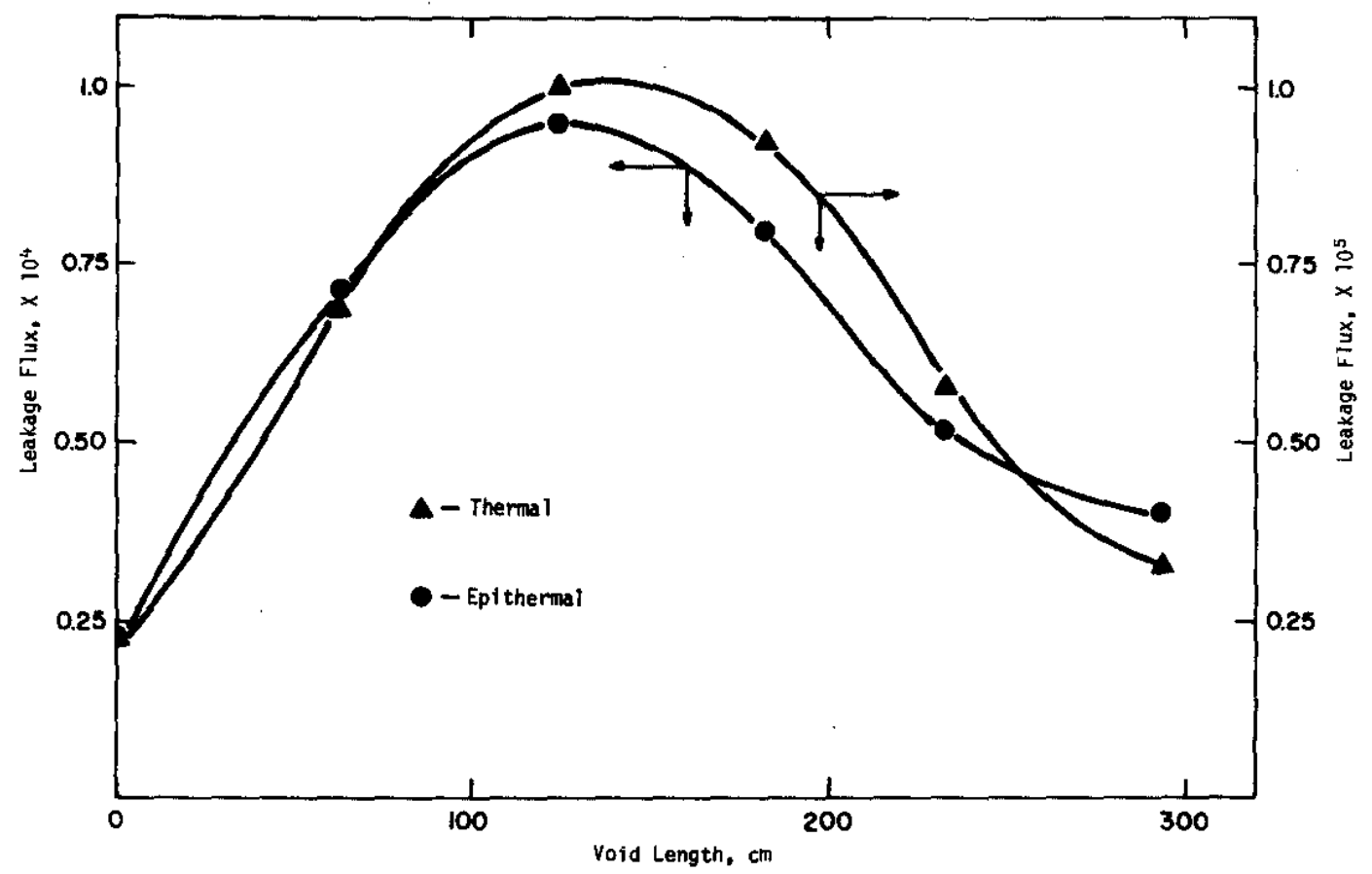

FIGURE 31. Measured Leakage Flux Data as a Function of Void Length - Uranium Metal Lattice 
was not established. This correction was accomplished by calculating with GLASS a supercel1 containing a vacant septifoil position in which reaction rates were computed for each resonance foil isotope at the proper cell position. Effective thermal reference cross sections were obtained from the relationship for a given foil $\mathrm{X}$ by

$\sigma_{X}^{e f f}=\frac{(\text { Reaction Rate) } X}{\left(\text { Reaction Rate) }{ }_{A u}\right.} \cdot g \sigma_{A u}^{2200}$

the reaction rates are from the GLASS edits

$\mathrm{g}$ is the Wescott $\mathrm{g}$ factor ${ }^{10}$

$\sigma_{\mathrm{Au}}^{2200}$ is the $2200 \mathrm{~m} / \mathrm{sec}$ gold neutron capture cross section

The resonance foil data were next corrected for isotopic abundance, thermal and resonance flux depression, and shielding effects of cadmium on the desired resonance. With this information, the resonance flux for a resonance foi $1 \mathrm{X}$ is computed by the following equation

$$
\phi_{X}^{\text {res }}=1 / f\left(A_{X}^{\text {Cd }} / A_{X}^{\text {ref }}\right)\left(F^{\text {th }} / F^{\text {res }}\right)\left[g \sigma_{X}^{\text {eff }} /(R . I .){ }_{X}\right]
$$

$1 / f$ is the cadmium resonance shielding factor

$\mathrm{A}_{\mathrm{X}}^{\mathrm{Cd}}$ is the cadmium-covered corrected activity for foil $\mathrm{X}$

$A_{X}^{r e f}$ is the reference activity for foil $X$

$\mathrm{F}^{\text {th }}$ and $\mathrm{F}^{\text {res }}$ are the thermal and resonance flux depression factors

$\mathrm{g} \sigma_{X}{ }^{\text {eff }}$ is the effective thermal reference cross section

(R.I.) $X$ is the infinite dilution resonance integral for
foil $X$

In the case of the ${ }^{6}{ }^{3} \mathrm{Cu}$ resonance, the flux must be computed with a resonance and a $1 / v$ smooth component. The $1 / v$ component is tied experimentally to a convenient low-energy resonance such as the ${ }^{115} \mathrm{In}$ resonance at $1.46 \mathrm{eV}$. Finally, the subcadmium resonances of ${ }^{3{ }^{9}} \mathrm{Pu}$ and ${ }^{176} \mathrm{Lu}$ require a special two-step procedure. Because these resonances are broad, the flux energy shape over 
the resonance must be used to estimate the flux at the resonance peak energy. This technique is described in detail in Reference 11. Basically, a flux shape guess (or computed estimate) is used in the first step to calculate the reaction rates over the resonance. Equal lethargy units are marked off on either side of the resonance centroid, and appropriate resonance cutoff points are selected. The differential cross section is numerically integrated over these two cutoff points to obtain the resonance integral, and the resonance flux is calculated as in the case of epicadmium resonances. The second step is to fit the spectrum computed by GLASS to a11 of the resonance flux data and use the resulting 37 energy group fluxes to recompute the reaction rate procedure. This results in an approximate 10 to $20 \%$ change in subcadmium resonance fluxes.

In the Enriched Lattice, three positions were monitored for spectral information. Figure 32 shows the spectrum at the center of the void region. Figure 33 is obtained from foils placed in the center of one of the void-region fuel assemblies, and Figure 34 is obtained from a location in the moderated region adjacent to the void (approximately $5 \mathrm{~cm}$ from the void interface). The striking feature of all these spectra is their similarity to a normally moderated spectrum which shows a thermal peak followed by a relatively flat ( $1 / E$ flux) dependence out to very high energies $(\sim \mathrm{MeV})$. The dashed curves in the figures represent the spectrum which results from a single cell or small supercell calculation. The solid curve is the normalized 37-energy group flux using the special 37-cell lattice pattern. The normalization point is at the gold $4.9 \mathrm{eV}$ resonance.

\section{Uranium Metal Lattice}

Similar data and analyses were obtained for the Uranium Metal Lattice and are illustrated in Figures 35-37. Note that no attempt has been made to assign uncertainties to the data. Because of the large number of data corrections and graphical treatment of subcadmium resonances, quantitative error analysis would be difficult. Best estimates without rigor indicate about \pm 25 to $30 \%$ uncertainty in the worst cases. Since the differences between calculated and experimental results span several orders of magnitude, these uncertainties are quite acceptable. Further refinement of data or experimental technique would have no meaningful effect on the computed void reactivity worths. 


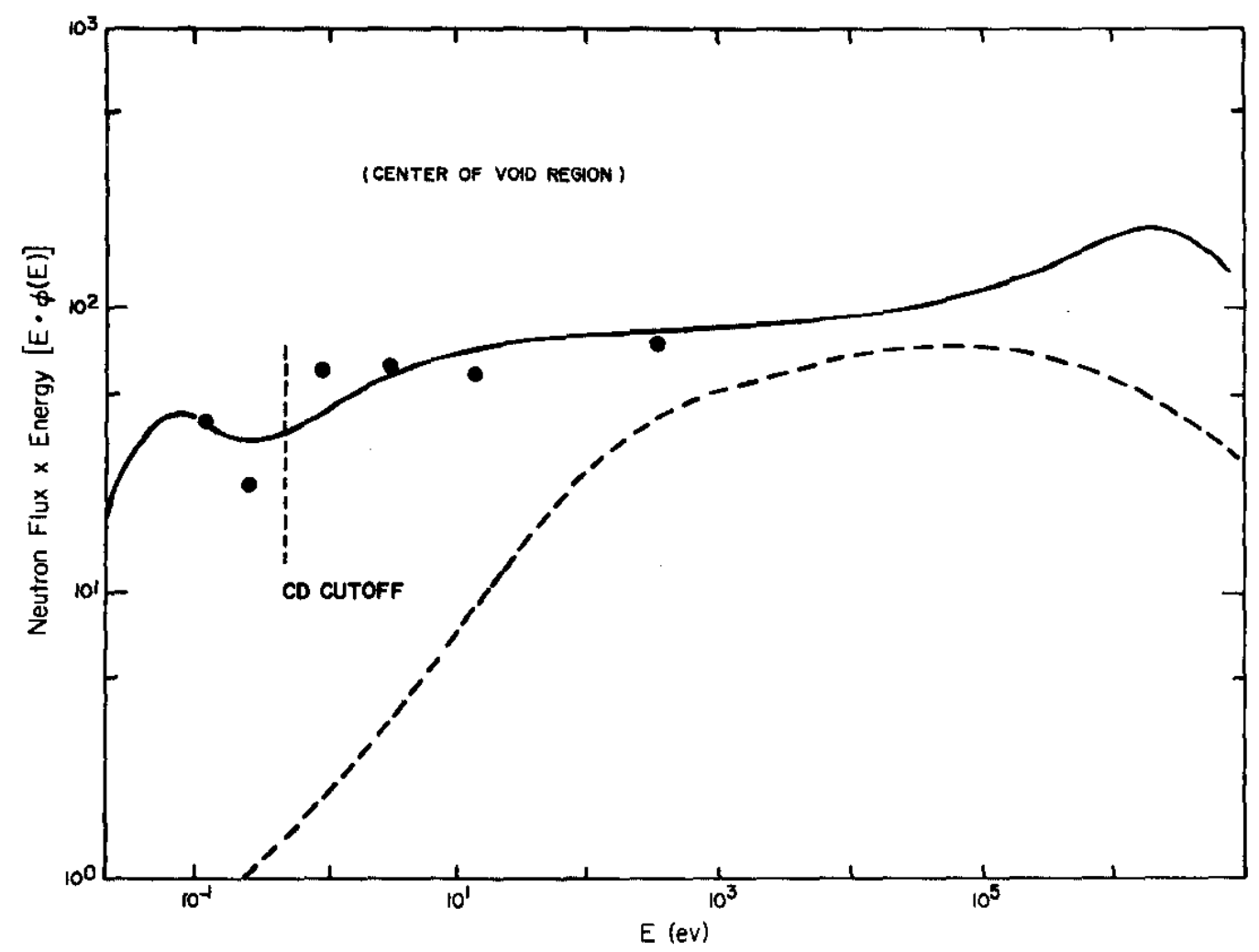

FIGURE 32. Comparison of Measured and Calculated Neutron Energy Spectra for the Enriched Lattice at the Center of the Void Region 


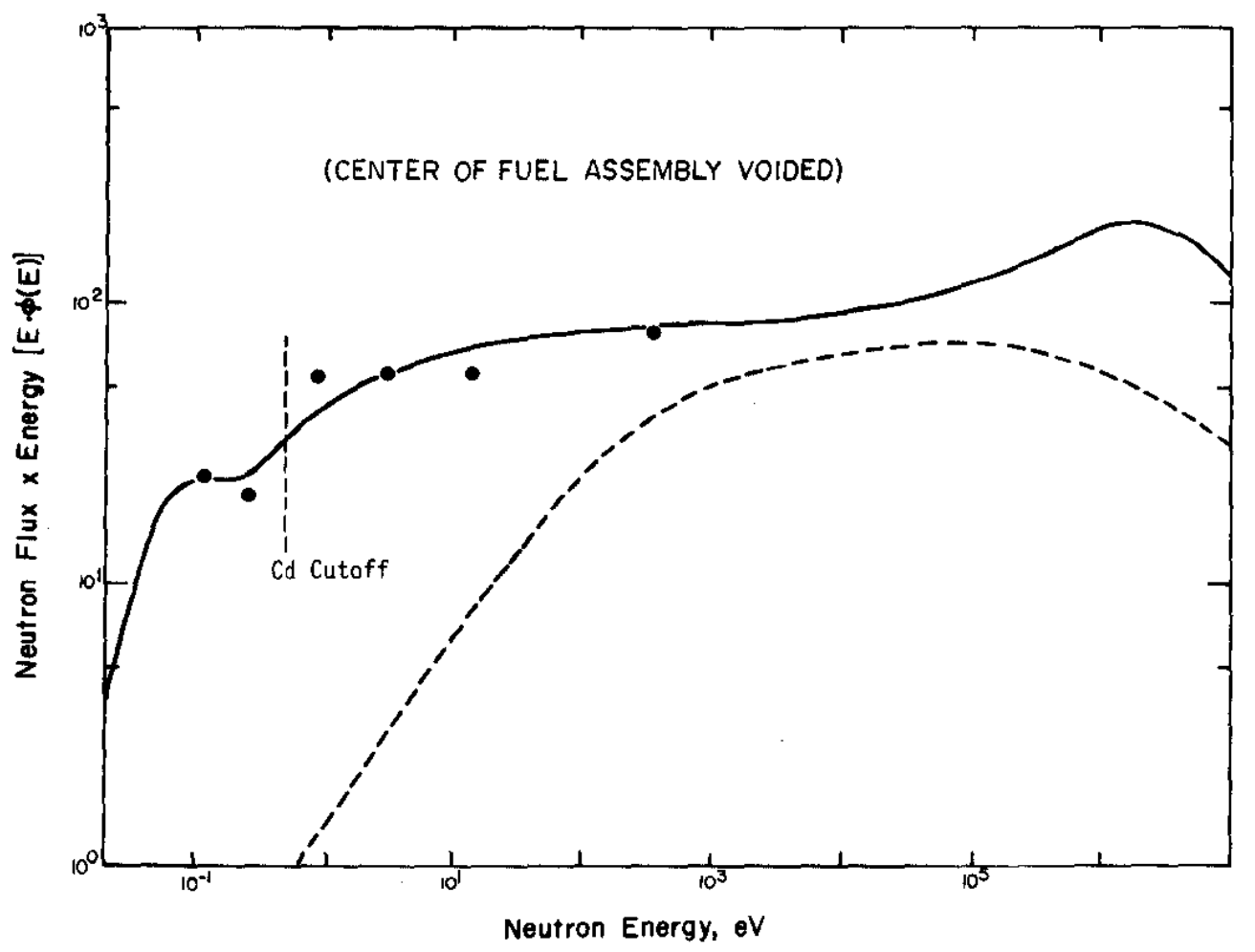

FIGURE 33. Comparison of Measured and Calculated Neutron Energy Spectra for the Enriched Lattice at the Center of a Voided Fuel Assembly 


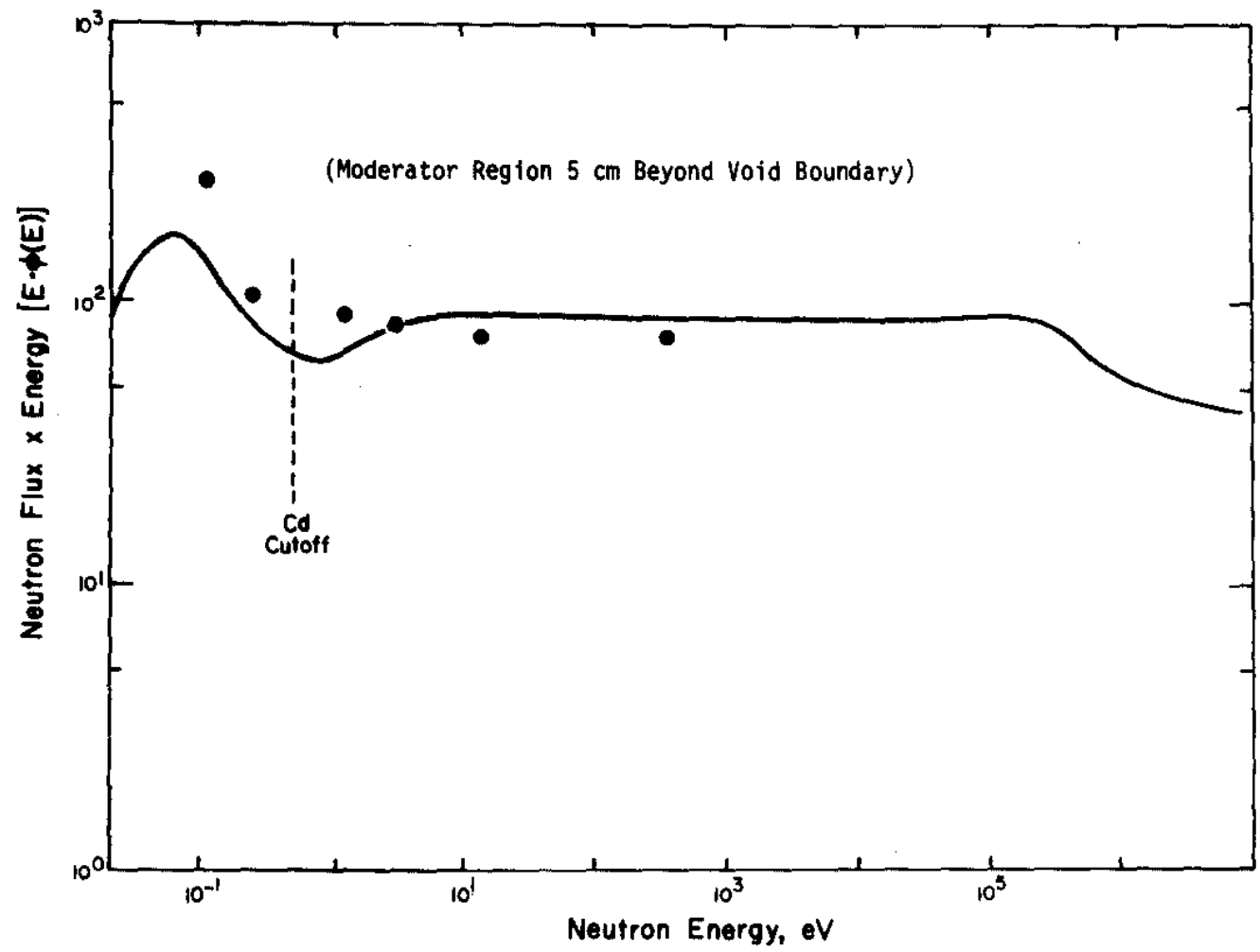

FIGURE 34. Comparison of Measured and Caiculated Neutron Energy Spectra for the Lattice $5 \mathrm{~cm}$ from Void Interface (in Moderator) 


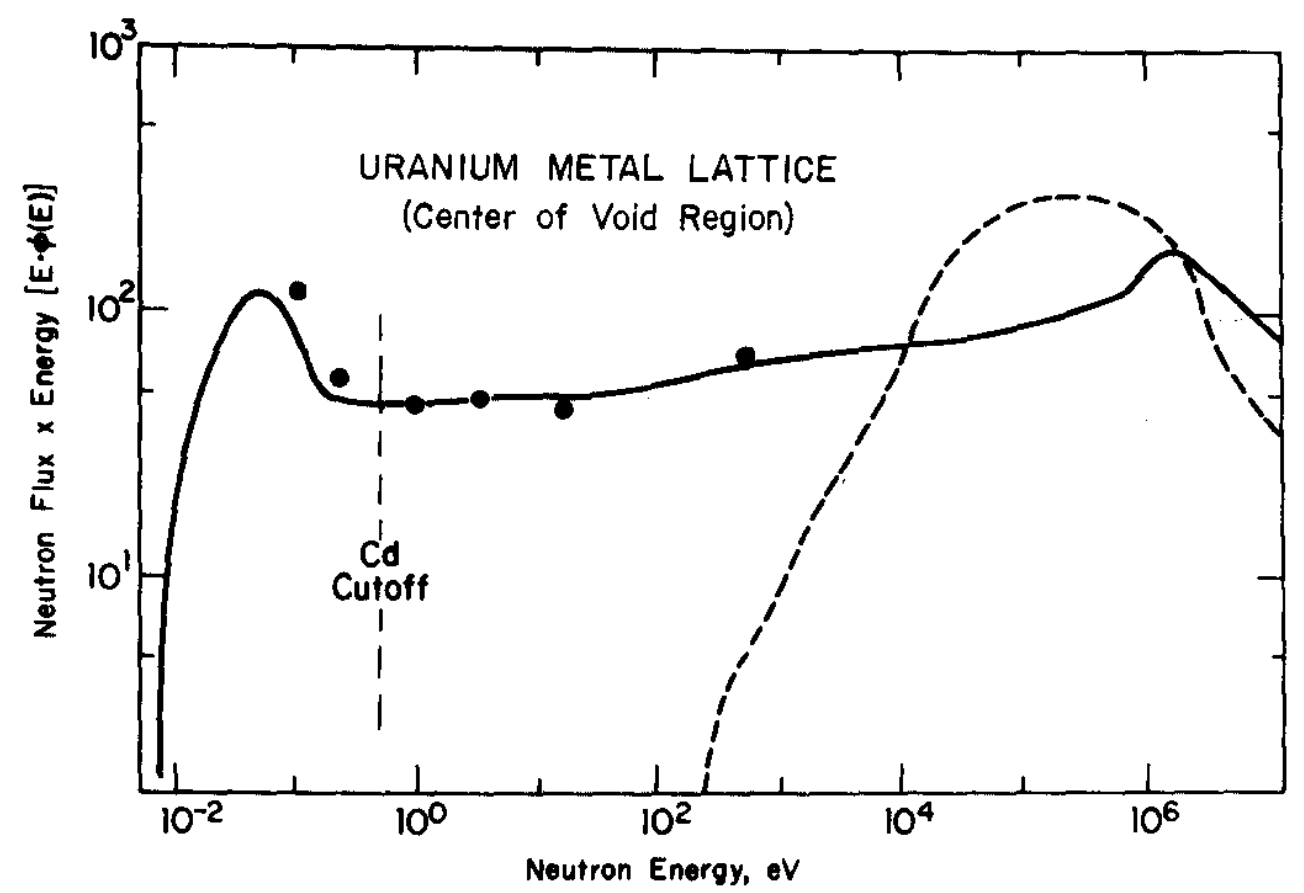

FIGURE 35. Comparison of Measured and Calculated Neutron Energy Spectra for the Uranium Metal Lattice at the Center of the Void Region 


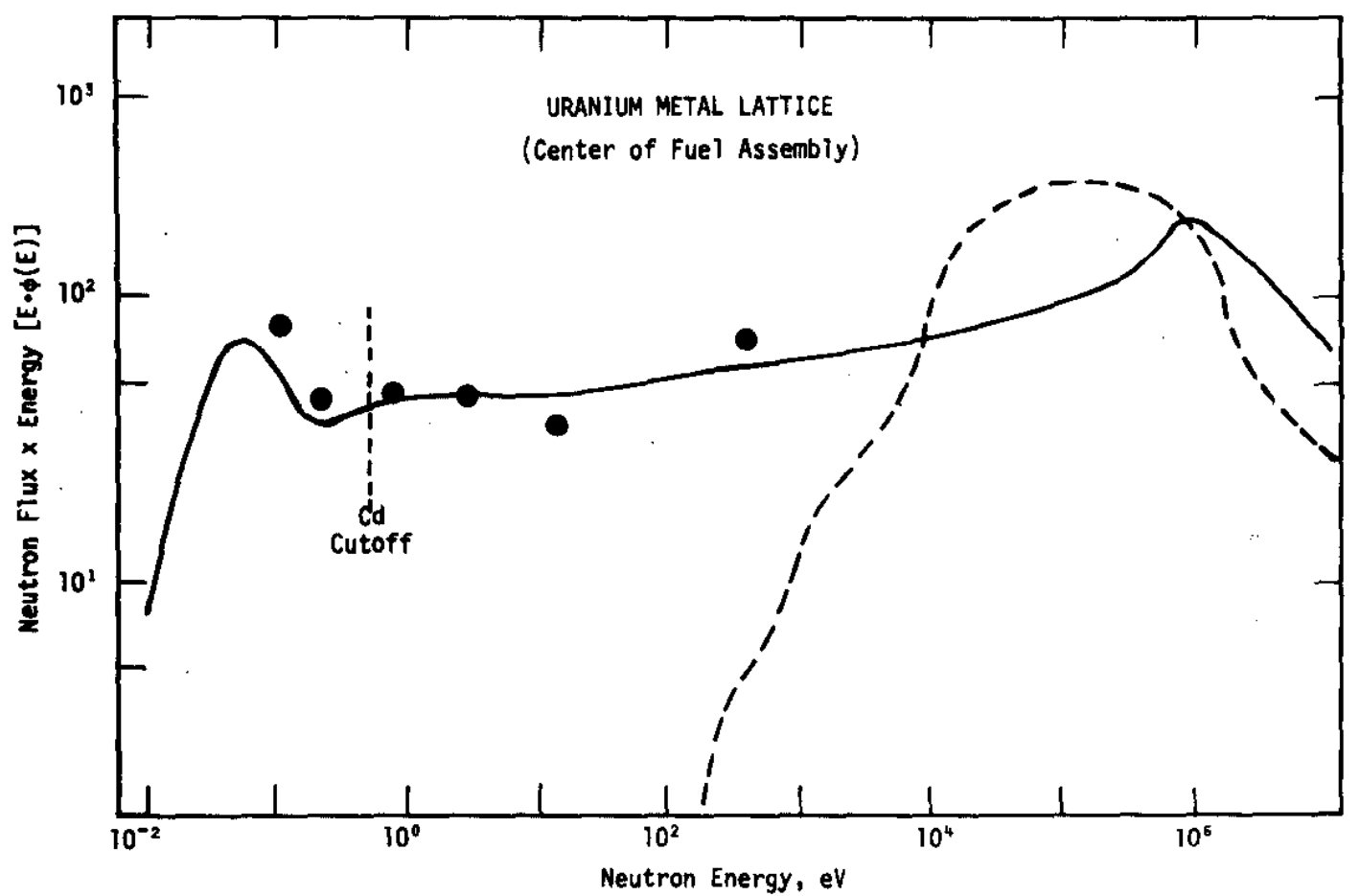

FIGURE 36. Comparison of Measured and Calculated Neutron Energy Spectra for the Uranium Metal Lattice at the Center of a Voided Fuel Assembly 


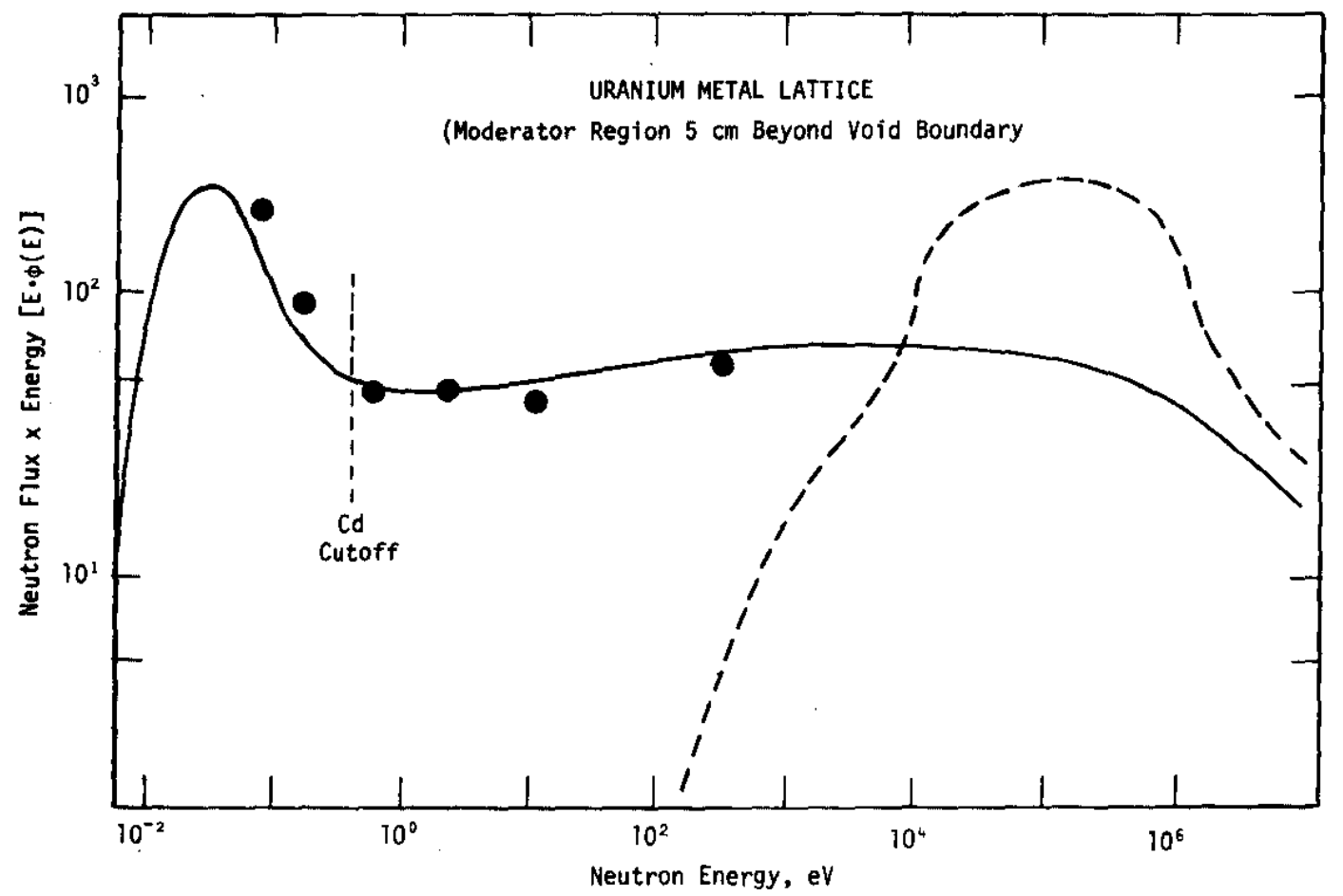

FIGURE 37. Comparison of Measured and Calculated Neutron Energy Spectra for the Uranium Metal Lattice $5 \mathrm{~cm}$ from Void Interface (in Moderator) 
Calculations of moderator void reactivity worths using standard lattice patterns and diffusion coefficients will result in a significant overestimate of the void worth; this is nonconservative for reactor safety analysis. However, by using a 37-ce11 lattice pattern and by computing diffusion coefficients either by the LEAK code or by Graves alternate method (see Appendix), good estimates of void worths will result.

Because the efficacy of the basic codes has been established for complete voiding of relatively large regions ( 7 assemblies), no further experiments of this particular type are deemed necessary at this time. However, other void-related questions are unresolved and should be reviewed for potential experimental evaluation. All experimental void studies to date have been dominated by neutron leakage out of the core. Therefore, calculational accuracy of void worth resulting from an embedded void in the core interior is still untested. Scoping calculations will be necessary to determine if this type of measurement is feasible and if the results of such a measurement will add to our understanding of void effects.

Another variation in void calculations is encountered when incomplete voiding occurs (i.e., mixed steam and liquid), and this effect has never been tested by controlled experiment at SRP/SRL. We are presently able to calculate with confidence reactivity worths for $0 \%$ and $100 \%$ void fractions. However, for intermediate void fractions, significant nonlinearities may exist. In fact, calculations of void worth as a function of void fraction show a rapid increase in void worth at higher void fractions. Experiments for fractional voiding are rather difficult, especially at high void fractions. 


\section{REFERENCES}

I. H. R. Reeve, J. A. Smith, H. E. Hootman, and W. S. Durant. "Loss-of-Target Analyses." Trans. Am. Mucl. Soc. 16, 208 (1973).

2. H. C. Honeck, R. J. Pryor, C. E. Henderson, and W. H. Hightower. "JoshuA Lattice Physics Subsystem." Proc. Conf. New Developments in Reactor Mathematics and Applications, March 29-31, 1971, Idaho Fal1s, Idaho. USAEC Report CONF-710302, Vo1. 2, pp 913-919 (1971). (Available from the National Technical Information Service, U.S. Department of Commerce, Springfield, Virginia 22151).

3. M. Michelini. "Anisotropic Diffusion Theory." Energia Nucleare 16 (11), 700 (1969).

4. H. C. Honeck and J. W. Stewart. "Simultaneous Line OverRelaxation (SLOR) in Hexagonal Lattice." Proc. New Developments in Reactor Physics and Shielding, September 12-15, 1972, Kiamesha Lake, New York, USAEC Report CONF-720901, Bk. 1, pp 253-265 (1972). (Available from the National Technical Information Service, U.S. Department of Commerce, Springfield, Virginia 22151)

5. P. Benoist, "Steaming Effects and Collision Probabilities in Lattices." NucZ. Sci. Eng. 34, 285 (1968).

6. R. J. Pryor and W. E. Graves, "Response Matrix Method for Treating Reactor Calculations," Proc. Mathematical Models and Computational Techniques for Analysis of Nuclear Systems, Apri1 9-11, 1973, Ann Arbor, Michigan. USAEC Report CONF730414-P2, P VII-179 (1973). (Available from the National Technical Information Service, U.S. Department of Commerce, Springfield, Virginia 22151)

7. R. J. Pryor. "Recent Developments in the Response Matrix Method." Advanced Reactors: Physics, Design and Economics, J. M. Kallfelz and R. A. Karam, Eds., p 169, Pergamon Press, New York, New York (1975).

8. R. J. Tuttle. "Delayed-Neutron Data for Reactor-Physics." Nucl. Sci. Eng. 56, 37 (1975).

9. J. P. Church. "Evaluation of Published Delayed-Neutron Parameters for ${ }^{2{ }^{35}} \mathrm{U}$ and ${ }^{235} \mathrm{U}$ Heavy Water Systems." Nucl. Sci. Eng. 43, 229 (1971). 
10. C. H. Westcott. "Effective Cross Section Values for We11Moderated Thermal Reactor Spectra," 3rd edition corrected. Atomic Energy of Canada Limited, CRRP-960, November 1, 1960.

11. J. D. Spencer and K. W. MacMurdo. Transplutonium Cross Sections Determined in a Resonance Spectrum Irradiation Assembly. USERDA Report DP-1311, Savannah River Laboratory, E. I. du Pont de Nemours and Co., Aiken, S.C. (1974). 


\section{APPENDIX}

Modified Benoist Method for Diffusion Coefficient Generation for Large Moderator Voids

\section{By: W. E. Graves, Savannah: River Laboratory}

Transverse leakage is treated in diffusion theory reactor calculations by introducing regionwise fictitious absorption cross sections equal to $\mathrm{DB}_{\mathrm{T}}{ }^{2}$ where,

$$
\bar{D}=\frac{1}{3} \frac{\sum \lambda_{i}^{\prime} \phi_{i} v_{i}}{\sum_{i} \phi_{i} v_{i}}
$$

Here $\lambda_{i}^{\prime}$ is a mean free path associated with a particular region. If voids are present and infinite medium properties are assigned, equation (I) will generally overestimate the negative reactivity change due to leakage. Benoist ${ }^{1}$ has introduced a more accurate transport theory method of obtaining average diffusion coefficients. The basic equation in isotropic form, is

$$
\bar{D}=\frac{1}{3} \frac{\sum_{j} \lambda_{i} \sum_{j} \phi_{j} v_{j} P_{i j}}{\sum_{j} v_{j}}
$$

where $\lambda$ is the infinite medium transport mean free path and $P_{i j}$ is the probability that a neutron originating in region $j$ will have its first collision in region $i$ (assuming uniform source strength in j). Benoist's application of equation (2) to a unit cell is generalized here to treat a few contiguous regions in the reactor. Equating expressions (1) and (2) provides a means for evaluating $\lambda_{i}^{\prime}$. For the balance of this derivation, a tworegion configuration will be assumed to simplify the mathematics. Also assuming the first region to be a void or "near void" region and the second to have normal moderation, leads to the approximation that $\lambda_{2}^{\prime}=\lambda_{2}$. All but one of the probabilities can be eliminated by the conservation relations;

$$
\begin{aligned}
& P_{11}+P_{21}=1 \\
& P_{12}+P_{22}=1
\end{aligned}
$$


and the reciprocity relation

$$
\begin{array}{lllllll}
\lambda_{1} & v_{2} & P_{12} & \lambda_{2} & v_{1} & P_{21}
\end{array}
$$

Since region 2 is thick enough that first flight transmission is negligible, the remaining probability factor $P_{1} 1$ may be replaced by $P_{C}$, the first flight collision probability. Solving for $\lambda_{1}^{\prime} / \lambda_{2}$ and using the approximation that $\phi_{1}=\phi_{2}$, it can be shown that

$$
D_{1} / D_{2}=\lambda_{1}^{\prime} / \lambda_{2}=1+\left(1-\frac{\lambda_{2}}{\lambda_{1}}\right)+\frac{\lambda_{1}}{\lambda_{2}}\left(1-\frac{\lambda_{2}}{\lambda_{1}}\right)^{2} \mathrm{P}_{\mathrm{c}}\left(\frac{\mathrm{r}}{\lambda_{1}}\right)
$$

For cylindrical geometry, including anisotropy, equation (5) becomes

$$
\mathrm{D}_{1}^{Z} / \mathrm{D}_{2}^{z}=1+\left(1-\frac{\lambda_{2}}{\lambda_{1}}\right)+\frac{\lambda_{1}}{\lambda_{2}}\left(1-\frac{\lambda_{2}}{\lambda_{1}}\right)^{2} \mathrm{P}_{\mathrm{C}}\left(\frac{\mathrm{r}}{\lambda_{1}}\right) \cdot \frac{3}{1+2\left(\mathrm{~T}_{\mathrm{r}} / \mathrm{T}_{Z}\right)}
$$

where $T_{r}$ and $T_{z}$ are graphed by Benoist ${ }^{1}$ and $D_{1}^{z}, D_{2}^{z}$ indicate an axial diffusion coefficient. Comparisons of calculations using equation (6) have been made to the transport theory calculations of module LEAK and were found to be slightly larger $(5-15 \%)$.

\section{Reference}

1. P. Benoist. "Streaming Effects and Collision Probabilities in Lattices." Nucl. Sci. and Eng. 34, 285-307 (1968). 